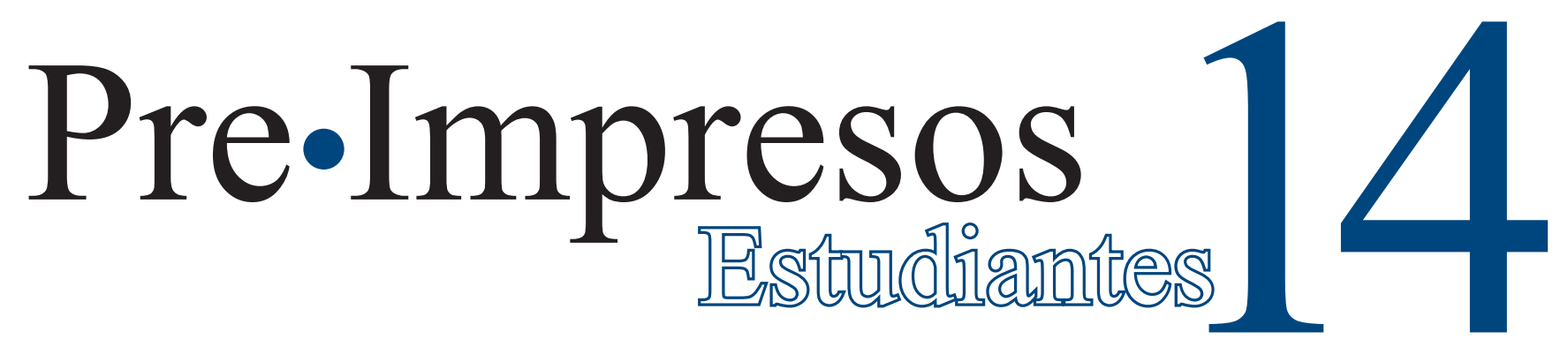

Facultad de Ciencia y Tecnología. Departamento de Tecnología - 2018-II • ISSN-E: 2323-0193 - ISSN 2539-0945

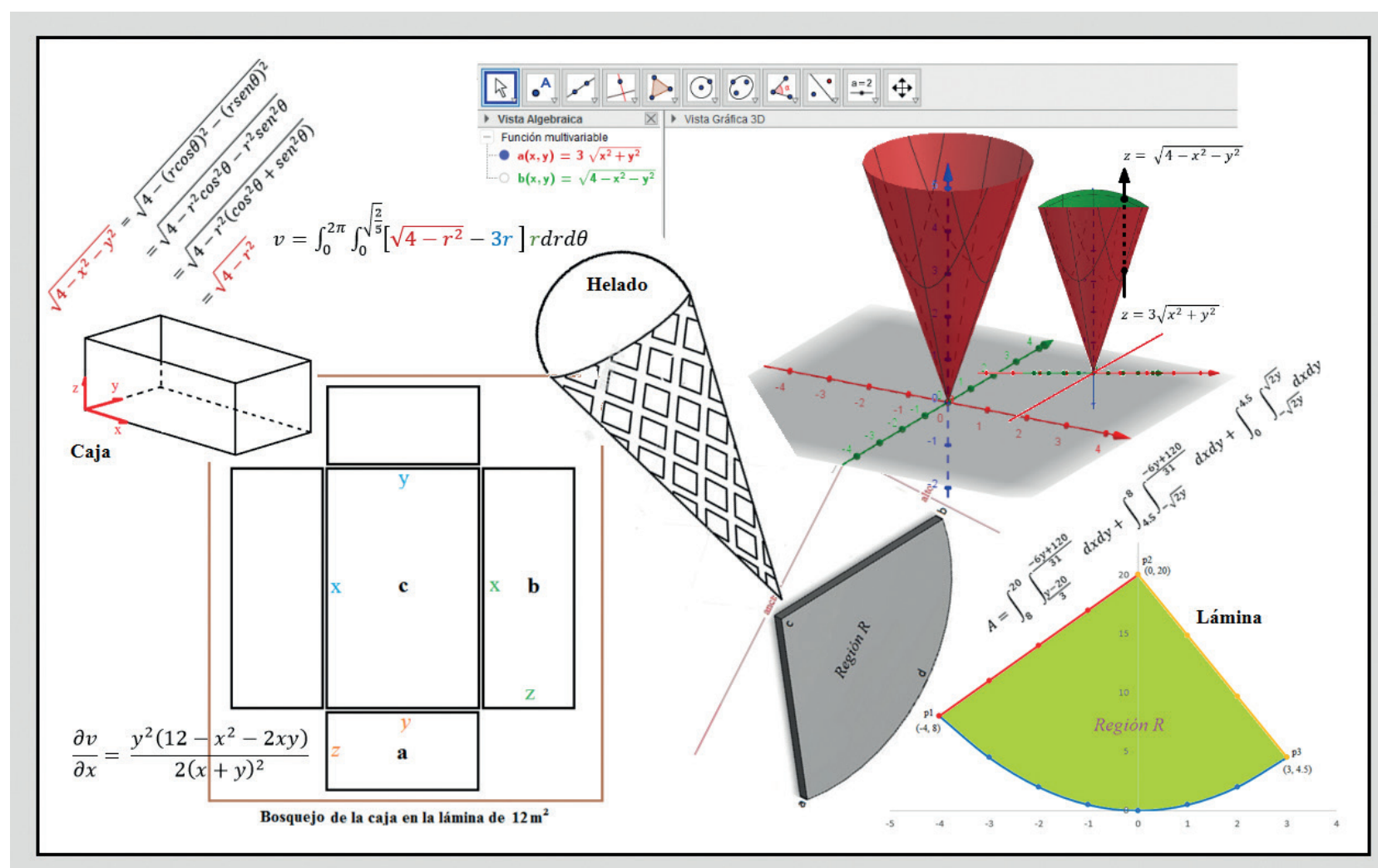

Extrapolación de situaciones de la vida real
a modelos matemáticos descritos mediante
derivadas parciales e integrales dobles Luis Fernando Maldonado Osorio Licenciatura en Electrónica 


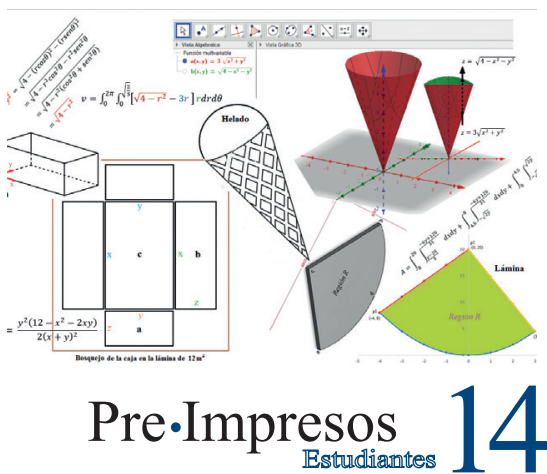

Leonardo Fabio Martínez Pérez Rector

Lyda Constanza Mora Mendieta Vicerrectora Académica

John Harold Córdoba Aldana Vicerrector de Gestión Universitaria

Fernando Méndez Díaz

Vicerrector Administrativo y Financiero

Helberth Augusto Choachí González

Secretario General

Facultad de Ciencia y Tecnología

Departamento de Tecnología

Nilson Genaro Valencia Vallejo

Director Departamento

Hugo Marín Sanabria

Coordinador Licenciatura en Electrónica

Yaneth Urrego Sierra

Supervisión de contenido

Juan Carlos Bustos Gómez

Director de la revista

Samuel Eduardo Sediles Martínez Coordinador de la revista

(C) Universidad Pedagógica Nacional

(C) Luis Fernando Maldonado Osorio

Portada e Imágenes

Luis Fernando Maldonado Osorio

Artículos publicados en diferentes medios escritos y referenciados en cada uno de los textos.

ISSN-E: 2323-0193

ISSN: 2539-0945

Diseño y Preparación editorial

Universidad Pedagógica Nacional

Grupo Interno de Trabajo Editorial 2018

Alba Lucía Bernal Cerquera

Coordinadora Grupo Interno

de Trabajo Editorial

Viviana Vásquez

Editora de Revistas

Impreso por

Xpress Estudio Gráfico y Digital S. A. S.

Bogotá, Colombia

\section{Extrapolación de situaciones de la vida real a modelos matemáticos descritos mediante derivadas parciales e integrales dobles}

Resumen

Diseño de una caja de cartón que ilustra el tránsito de área a volumen

Cálculo del área de la superficie de una pieza mecánica

Cálculo del área de la superficie plana de una pieza

en un sistema excéntrico

Cálculo del volumen de un helado en un instante de tiempo

Cálculo de la masa de una lámina de densidad no homogénea

Notas complementarias

Referencias

\section{Presentación}

La serie Pre.Impresos Estudiantes es una iniciativa editorial del Proyecto Comunicación y Publicaciones de la Facultad de Ciencia y Tecnología (FCT), cuya idea central es trabajar por la cualificación de la escritura, para dar visibilidad a la producción intelectual de los maestros en formación y en ejercicio. Con esta publicación se busca tender puentes entre los saberes especializados y la cultura en general, además de contribuir al fortalecimiento de la docencia y la investigación en educación.

Asimismo, constituye una estrategia de comunicación que posibilita la circulación adecuada de información y promueve la reflexión sobre temas y actividades inherentes a las ciencias, la matemática, la tecnología y su enseñanza. Con ella también se espera favorecer la integración de los equipos de trabajo y la construcción de relaciones de cooperación entre los diferentes miembros de la comunidad académica de la Facultad.

Estos aspectos, relacionados con los fines misionales de la Universidad Pedagógica Nacional, resultan pertinentes y significativos en la formación de nuevas generaciones de maestros e investigadores en pedagogía, que en su futura práctica profesional afrontarán diversos retos y circunstancias que el entorno social del país le plantea a la educación.

Información:

pre_impresos@pedagogica.edu.co

Facultad de Ciencia y Tecnología

Teléfonos: (57) (1) 3471190 / 5941894 Ext. 242 


\title{
Extrapolación de situaciones de la vida real a modelos matemáticos descritos mediante derivadas parciales e integrales dobles
}

\author{
Luis Fernando Maldonado Osorio \\ dte_Ifmaldonadoo358@pedagogica.edu.co
}

\section{Resumen}

Este trabajo invita al lector a extrapolar situaciones de la vida real a modelos matemáticos, descritos mediante derivadas parciales e integrales dobles para comprender la esencia del cálculo multivariado. Asimismo sirve como una herramienta que impulsa al analisis de innumerables situaciones y su posterior modelamiento matemático.

Los ejercicios propuestos sobre situaciones cotidianas utilizan procesos metodológicos que incluyen los contextos situacionales en los que las personas exploran, experimentan y aprenden a diario. Se proponen cinco ejercicios que se desarrollan paso a paso, utilizando gráficas, expresiones literales y numéricas. La primera situación tiene como propósito determinar el volumen máximo que puede tener una caja de cartón, conociendo solo la dimensión de la lámina y el bosquejo de la caja, para hacer la tran- sición de área a volumen se utiliza una ecuación matemática basada en las partes de la caja. En la segunda y tercera situación, se busca hallar el área de una superficie plana de una pieza mecánica, teniendo en cuenta diferentes conceptos con el fin de construir un sistema excéntrico y un molde para la inyección de plástico. En la cuarta situación, se determina el volumen de un helado que saborea una persona en un instante de tiempo. En el quinto ejercicio situacional, que se desarrolla en el contexto de la industria radiológica, se cálcula la masa de una lámina de densidad no homogénea, para garantizar un excelente blindaje a los rayos ultravioleta y rayos gama. Por ultimo, se presenta un compendio de conceptos necesarios para la comprensión de cada situación-problema.

Palabras clave: Modelamiento matemático, integrales dobles, derivadas parciales, suma de Riemann, sólidos de revolución 


\section{Abstract}

The current proposal encourages the readers to extend different real life situations to mathematical modelling, which can be described by partial derivatives and double integrals to grasp the most relevant concepts of multivariable calculus. In that respect, this can be used as tool to analyze another countless situations and subsequent mathematical modelling.

Several exercises have been proposed, focusing on daily situations and using methodological processes, including situational context, in which people explore, experience and learn each day. Hence, five exercises are suggested and developed step by step, showing graphics, and using literal and numeric expressions. The first exercise shows a problematic situation, in which is necessary to determine what maximum volume can have a paperboard box, considering that is only known the size of the sheet and the sketch of the box. Therein, it is possible to propose a

\section{Introducción}

En la presente cartilla se destaca la relación existente entre el mundo real y su representación matemática, se analizan un conjunto de situaciones cotidianas, estudiadas y descritas mediante un modelo, ya sea, conceptual, gráfico o matemático, lo que hace posible comprender dichas situaciones para tomar decisiones y optimizar recursos que lleven a la máxima eficiencia, esto mediante el registro de la mayor cantidad de variables posibles para relacionarlas, a través del desarrollo de derivadas parciales e integrales de varias variables, de manera que garanticen proximidad a la realidad.

En este sentido, se observa que la modelación matemática de una acción tan sencilla como la de saborear un helado nos permite entender el estado de esa acción en un momento determinado. Como estudiante he podido vivenciar que la matemática enseñada de forma descontextua- mathematical description based on the parts of the box to determine the transition from area to volume. The second and third exercise require the calculation of the area of a flat surface of certain mechanical piece considering different concepts to build an eccentric system and a mold for plastic injection.

In the fourth exercise is requested the volume of an ice cream container, which a person is tasting in a certain time. The last exercise is a typical problematic situation in the radiology, in which is determined the mass of sheet with inhomogeneous density to assure an excellent shielding of ultraviolet and gamma rays. Finally, this proposal introduces a review of the most important concepts needed to understand each mentioned situation.

Keywords: Mathematical modelling, double integrals, partial derivatives, Riemann sum, solid of revolution

lizada limita la comprensión de los conceptos y conlleva un aprendizaje netamente memorístico de pasos repetitivos, que no requiere entender la esencia de lo que representan los ejercicios. Asimismo, el conocimiento de las matemáticas va más allá de lo que usualmente ilustran los textos de estudio, cómo es el caso de una ecuación que debe ser resuelta privilegiando únicamente el proceso operativo y mecánico, sin extrapolar lo que podría representar la misma ecuación en la cotidianidad.

Esto ha dado origen a la creencia de que la matemática es un cuerpo rígido, de fórmulas y verdades absolutas que no da espacio a la imaginación y a la creatividad propositiva. Esta visión hermética, inaccesible y velada dificulta ver otros posibles caminos para comprenderla e induce a pensar que es una disciplina restringida a unos pocos. 
Teniendo en cuenta lo anterior, se desarrolló un enfoque didáctico que puede ser implementado como una estrategia pedagógica para enseñar ciertas temáticas del cálculo usando situaciones prácticas, puesto que permite que los estudiantes interesados en el contenido de la cartilla desarrollen habilidades de pensamiento a la hora de argumentar, identificar, ejemplificar, clasificar y demostrar la solución que puedan construir de una determinada situación problema desde los mismos contextos en los que viven.

Todas estas situaciones se desarrollan con representaciones gráficas a fin de brindar al lector variadas herramientas que podrá aplicar en diferentes contextos, obteniendo como resultado modelos basados en derivadas parciales o integrales dobles que reflejan matemáticamente cada uno de los contextos. En suma, esta propuesta es muy útil en la práctica y constituye una invitación para que el lector aborde el estudio del cálculo multivariado, pues emplea la estrategia didáctica de relacionar lo simbólico con lo real, contribuyendo a mejorar la transposición de los conceptos y su comprensión. Igualmente, invita a que el lector avive la capacidad para visualizar, diseñar e interpretar fenómenos, fortaleciendo la autonomía y el auto aprendizaje de manera significativa.

Asimismo, la cartilla en cada una de sus secciones presenta los ejercicios partiendo de lo simple hasta llegar a lo complejo y utiliza una didáctica situacional de manera que permita valorar la aplicación de las matemáticas en la cotidianidad. Esta ha sido diseñada y dirigida a personas que tengan un conocimiento básico de los métodos de derivación e integración de funciones de una sola variable.

Por último, quiero agradecer a todas las personas que hicieron posible esta publicación, al equipo de trabajo del proyecto Pre-Impresos por apoyar la difusión del conocimiento de profesores y estudiantes de las diferentes licenciaturas de la FCT para promover el aprendizaje de las personas $y$, en particular, al profesor Juan Carlos Bustos, pues, sin lugar a duda, el trabajo final es un reflejo de su constante acompañamiento. De igual modo, les recuerdo a los lectores que este documento también está disponible en la plataforma Open Journal System de la Universidad, desde donde se puede descargar en formato digital.

\section{Diseño de una caja de cartón que ilustra el tránsito de área a volumen}

En una fábrica de cajas de cartón tienen destinado $144 \mathrm{~m}^{2}$ de papel kraft para producir cierta cantidad de unidades. Un cliente solicitó que se diseñaran sin tapa, como se muestra en la imagen 1. Después de realizar un estudio acerca de la cantidad de papel kraft que se utilizará en su elaboración, el departamento de procesos concluyó que se necesitará una lámina de $12 \mathrm{~m}^{2}$ para elaborar cada caja. Si quisiéramos determinar el número total de cajas que se pueden construir con los $144 \mathrm{~m}^{2}$ de papel, se necesitaría conocer de antemano el volumen máximo que se obtiene con la lámina de $12 \mathrm{~m}^{2}$ de papel kraft destinado para cada una de las cajas.

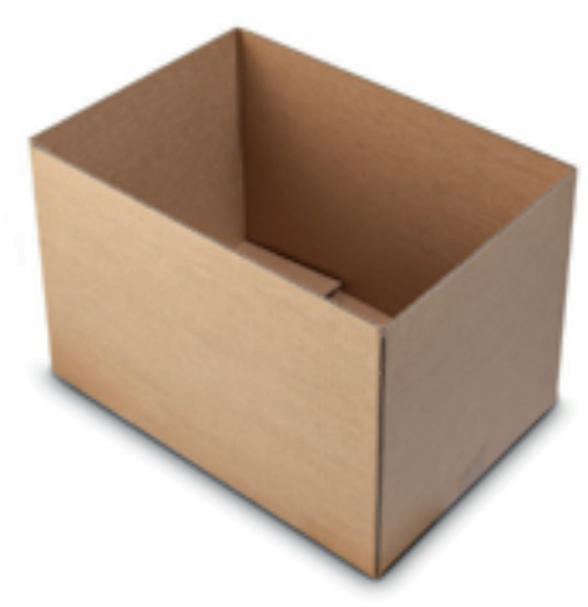

Imagen 1. Caja de cartón

Fuente: https://casaimagenes.info/cajas-carton/cajascarton-elegant-caja-sin-solapas-a-medida/ 
Para dar respuesta a la pregunta anterior, tenemos que identificar con qué información contamos. El ejercicio plantea lo siguiente:

Se tienen destinados $144 \mathrm{~m}^{2}$ de papel kraft para construir cierta cantidad de cajas y en cada una se utilizarán $12 \mathrm{~m}^{2}$. Además, nos dicen que las cajas se diseñarán sin tapa. Esto implica que solo tendrán cinco caras, lo que constituye la primera condición para diseñarlas.

Entonces, para determinar el volumen final de cada caja y el número total de cajas que se pueden fabricar con la lámina de $144 \mathrm{~m}^{2}$, podemos recurrir a varios métodos, en este caso utilizaremos el cálculo para dar respuesta a estas interrogantes.

Así, con la información disponible, se plantean las ecuaciones que relacionan las condiciones o restricciones.
Tomamos como punto de referencia uno de los vértices de la caja para ubicar los ejes $x, y y$ $z$, siendo:

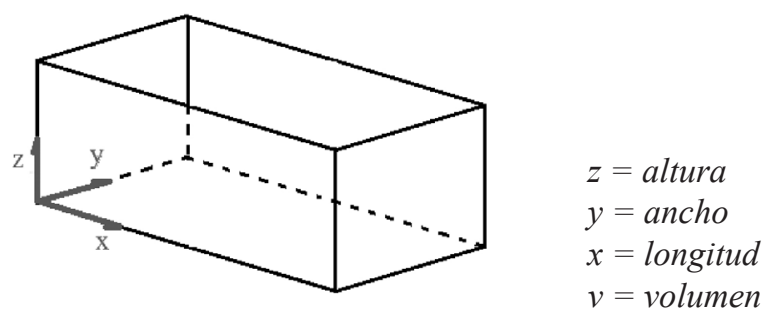

Figura 1. Volumen de la caja

Por tanto, el volumen se representa de la siguiente forma:

$$
V=z * y * x
$$

La ecuación (1.a) se explica gráficamente de la siguiente manera:

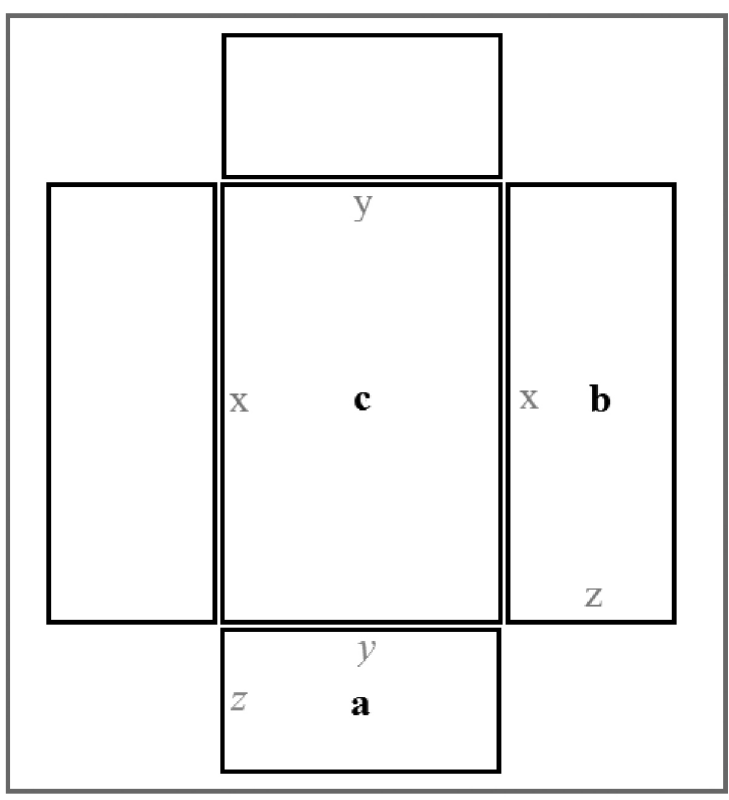

Bosquejo de la caja en la lámina de $12 \mathrm{~m}^{2}$

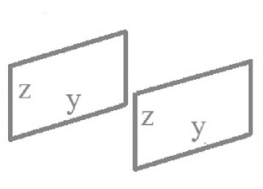

a.

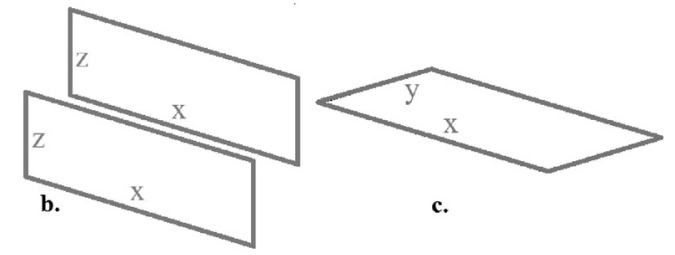

Figura 2. Bosquejo de la caja en la lámina de $12 \mathrm{~m}^{2}$ 
Las imágenes $\mathbf{a}$, b y c corresponden a las diferentes caras de la caja. Cada cara es la unión de la proyección de dos ejes. Si observamos, por ejemplo, la imagen a evidenciamos la proyección de los ejes $z$ y y, de igual manera, la imagen $\mathbf{b}$ y $\mathbf{c}$ muestran la proyección de los ejes $z$ y $x$, y de los ejes y y $x$, respectivamente.

Luego de haber realizado un desglose gráfico para identificar las variables que forman cada cara de la caja, se procede a relacionar estos datos matemáticamente.

Tenemos una lámina (bidimensional) de 12 $\mathrm{m}^{2}$ con la cual se construirá una caja (tridimensional). Es decir, si se quiere optimizar el uso del material, para minimizar las pérdidas, el volumen máximo que se puede obtener equivaldrá a los $12 \mathrm{~m}^{2}$. Esto se expresa como $V_{\text {máximo de la caja }}$ conformado por la altura (z), la longitud ( $\mathrm{x}$ ) y el ancho (y).

De lo anterior obtenemos la siguiente igualdad.

$$
12 \mathrm{~m}^{2}=V_{\text {máximo de la caja }}
$$

Las caras de la caja siempre estarán descritas por un par de variables (yx, zx, yz), porque cada cara se representa como un rectángulo formado por dos dimensiones (véase la Figura 2).

Por tanto, la ecuación que relaciona las 5 caras de la caja y la lámina de papel kraft es:

$$
12 \mathrm{~m}^{2}=y x+2 z x+2 y z
$$

En la ecuación (1.b) se observa como yx representa la cara que forma la base de la caja, $2 z x$ representa las dos caras laterales derecha e izquierda y $2 y z$ representa las dos caras frente y posterior. La suma de las caras de la caja es igual a los $12 \mathrm{~m}^{2}$ de papel kraft.

La ecuación (1.b) permite relacionar las dimensiones de cada cara de la caja con la ecuación (1.a) del volumen. Aunque se parte de una lámina de dos dimensiones, como se habla de construir una caja de tres dimensiones, implícitamente se está hablando de volumen.

Se plantea la ecuación (1.b) en términos de dos variables para sustituirla en la ecuación (1.a).

Para este caso se despejará la variable $z$ de la ecuación (1.b), z en la lámina representa un lado de la cara a y b., también se hubiera podido despejar $x$ o y conduciendo a la misma solución.

$$
y x+2 z x+2 y z=12 \mathrm{~m}^{2}
$$

Se factoriza

$$
z(2 x+2 y)=12-y x
$$

Al despejar z en la ecuación (1.b) se obtiene:

$$
z=\frac{12-y x}{2 x+2 y}
$$

Se sustituye la ecuación (1.c) en la ecuación (1.a), con el fin de expresar el volumen en términos de dos incógnitas.

$$
\begin{aligned}
& V=z * y * x \\
& V(x, y)=\left(\frac{12-y x}{2 x+2 y}\right) y x
\end{aligned}
$$

Nótese que en esta parte del proceso se pasa de dos a tres dimensiones. Ahora, se hallarán las dimensiones de longitud $(x)$ y el ancho $(y)$.

Aplicamos la propiedad distributiva de la multiplicación y obtenemos la siguiente expresión:

$$
V(x, y)=\frac{12 y x-y^{2} x^{2}}{2 x+2 y}
$$


Se deriva parcialmente la ecuación (1.d) para obtener el valor numérico de $x$ y $y$, aplicando el método de derivación del cociente. ${ }^{1}$

A continuación se observa la derivada parcial respecto a $x$ de la ecuación (1.d)

$$
\begin{aligned}
& \frac{\partial V}{\partial x}=\frac{\left(12 y-2 x y^{2}\right)(2 x+2 y)-2\left(12 x y-x^{2} y^{2}\right)}{(2 x+2 y)^{2}} \\
& \frac{\partial V}{\partial x}=\frac{2\left(12 y-2 x y^{2}\right)(x+y)-2\left(12 x y-x^{2} y^{2}\right)}{4(x+y)^{2}} \\
& \frac{\partial V}{\partial x}=\frac{\left(12 y-2 x y^{2}\right)(x+y)-\left(12 x y-x^{2} y^{2}\right)}{2(x+y)^{2}}
\end{aligned}
$$

Se desarrolla el producto de los términos y operamos

$$
\frac{\partial V}{\partial x}=\frac{\left.12 x y+12 y^{2}-2 x^{2} y^{2}-2 x y^{3}-12 x y+x^{2} y^{2}\right)}{2(x+y)^{2}}
$$

De la ecuación anterior se despeja $y^{2}$ que corresponde al término común

$$
\frac{\partial V}{\partial x}=\frac{y^{2}\left(12-x^{2}-2 x y\right)}{2(x+y)^{2}}
$$

Ahora, para obtener el valor numérico del ancho, calculamos la derivada parcial respecto a y de la ecuación (1.d) siguiendo los mismos pasos anteriores,

1 La solución a un problema de optimización que comprende una función de varias variables exige hallar el extremo absoluto de la función. En este caso, el volumen que corresponde a nuestra función es expresado en términos de dos variables, lo cual permitirá hallar el máximo absoluto calculando la derivada parcial del volumen respecto a $x y y$.

$$
\begin{gathered}
\frac{\partial V}{\partial y}=\frac{\left(12 x-2 y x^{2}\right)(2 x+2 y)-2\left(12 x y-x^{2} y^{2}\right)}{(2 x+2 y)^{2}} \\
\frac{\partial V}{\partial y}=\frac{2\left(12 x-2 y x^{2}\right)(x+y)-2\left(12 x y-x^{2} y^{2}\right)}{4(x+y)^{2}} \\
\frac{\partial V}{\partial y}=\frac{\left(12 x-2 y x^{2}\right)(x+y)-\left(12 x y-x^{2} y^{2}\right)}{2(x+y)^{2}} \\
\frac{\partial V}{\partial y}=\frac{12 x y+12 x^{2}-2 x^{2} y^{2}-2 y x^{3}-12 x y+x^{2} y^{2}}{2(x+y)^{2}}
\end{gathered}
$$

De la ecuación anterior despejamos $x^{2}$ que corresponde al término común

$$
\frac{\partial V}{\partial y}=\frac{x^{2}\left(12-y^{2}-2 x y\right)}{2(x+y)^{2}}
$$

De las ecuaciones (1.e) y (1.f) se toma el término que está en el numerador entre paréntesis, al igualar los dos términos a cero se obtiene que $x^{2}=y^{2}$, por tanto $x=y$, esta igualdad permite reescribir la ecuación (1.e) solamente en términos de $x^{2}$ o en su defecto reescribir la ecuación (1.f) únicamente en términos de $y^{2}$.

$$
\left\{\begin{array}{l}
12-2 x y-x^{2}=0 \\
12-2 x y-y^{2}=0
\end{array}\right.
$$

Sustituyendo y por $x$ en la ecuación (1.g), hallamos que:

$$
\begin{gathered}
12-2 x^{2}=x^{2} \\
3 x^{2}=12
\end{gathered}
$$




\section{Pre.Impresos 14}

De modo que $x^{2}=4$.

Cada lado de la caja debe ser positivo, porque no existen magnitudes negativas, entonces el valor de $x=y=2$.

Para obtener el valor de $z$ (altura de la caja) se evalúa en la ecuación (1.c):

$$
z=\frac{12-y x}{2 x+2 y}=\frac{12-4}{8}=1
$$

Así, las dimensiones que maximizan ${ }^{2}$ el volumen de la caja son: $x=2, y=2$ y $z=1$.

\section{Cálculo del área de la superficie de una pieza mecánica}

El siguiente ejercicio tiene como objetivo determinar el área de una superficie plana en una pieza.

Se iniciará graficando las ecuaciones que describen la región acotada, estas ecuaciones se obtuvieron previamente parametrizando la cara de la pieza ${ }^{3}$, luego se identificarán los límites de la integral doble y, por último, se solucionará dicha integral. En seguida, se plantea la situación a analizar:

A una fábrica de inyección de plástico llega una persona que solicita el diseño de un molde para la siguiente pieza.

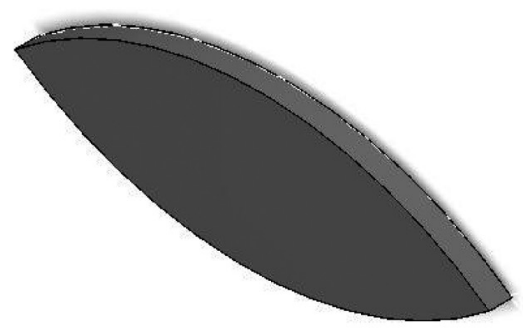

Figura 3. Pieza 1

Para mecanizar el molde se necesita conocer el área de la superficie plana de la pieza. Con este fin los ingenieros parametrizaron la pieza 1 y obtuvieron la siguiente región:

$$
R=\left\{(x, y) \mid x \geq y^{2}-4 y \cap x \leq 8-y^{2}\right\}
$$

A continuación, se grafican los intervalos que definen las funciones que acota la región $R$.

Para realizar la gráfica $x=y^{2}-4 y$ tabularemos 14 puntos desde -3 hasta 10, de igual manera se tomarán 11 puntos desde -5 hasta 5 para graficar la ecuación $x=8-y^{2}$. Se seleccionaron estos rangos con el fin de visualizar de manera óptima la región $R$ formada por las dos gráficas.

Existen varios métodos para graficar la función, pero se utiliza el método de tabulación para facilitar la comprensión del lector.

2 Es importante mencionar que una función continua definida en un intervalo arbitrario no siempre tiene un máximo absoluto; sin embargo, con frecuencia en las aplicaciones prácticas se garantiza la existencia del máximo absoluto de una función.

3 Parametrizar hace referencia a tomar la superficie de la pieza a trabajar y expresarla por medio de ecuaciones en el plano cartesiano. 


\begin{tabular}{cc}
\hline$y$ & $f(y)$ \\
\hline-3 & 21 \\
-2 & 12 \\
-1 & 5 \\
0 & 0 \\
1 & -3 \\
2 & -4 \\
3 & -3 \\
4 & 0 \\
5 & 5 \\
6 & 12 \\
7 & 21 \\
8 & 32 \\
9 & 45 \\
10 & 60 \\
\hline
\end{tabular}

Tabla a. $x=y^{2}-4 y$

$$
\begin{array}{r}
x=y^{2}-4 y \\
x(-3)=(-3)^{2}-4(-3) \\
x(-3)=9+12 \\
x(-3)=21 \\
x=8-y^{2} \\
x(-5)=8-(-5)^{2} \\
x(-5)=8-25 \\
x(-5)=-17
\end{array}
$$

Luego de obtener los valores de las dos ecuaciones se ubica cada pre-imagen (valor asignado a la variable independiente) con su respectiva imagen (valor obtenido al evaluar la pre-imagen en la ecuación), para unir cada punto por medio de una línea. De esta manera, se evidencia la región $R$ que se encuentra acotada por las dos parábolas horizontales $x=y^{2}-4 y$ y $x=8-y^{2}$, tal como se puede observar en la Gráfica 2a. ecuaciones, pues los demás punto para las se determinan de la misma manera.

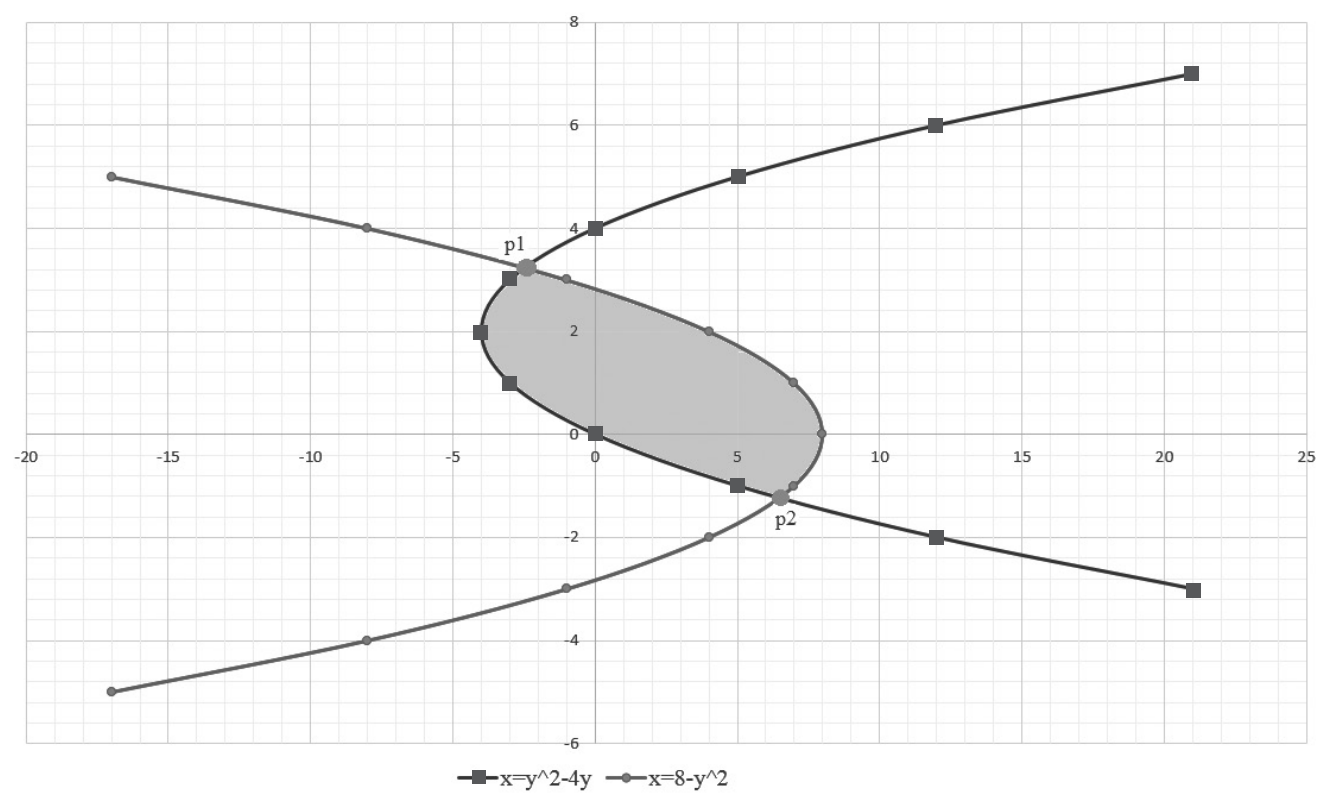

Gráfica 2a. Región $R$ 
Después, se iguala la ecuación $x=y^{2}-4 y$ con la ecuación $x=8-y^{2}$ para determinar los puntos de intersección (punto p1 y p2 de la gráfica 2a.)

$$
\begin{aligned}
y^{2}-4 y & =8-y^{2} \\
2 y^{2}-4 y-8 & =0
\end{aligned}
$$

Para obtener los puntos de intersección o raíces de la ecuación (2.c) se utiliza la ecuación cuadrática.

$$
a y^{2}+b y+c=0 \quad(a \neq 0)
$$

Las soluciones de la ecuación cuadrática están dadas por:

$$
y=\frac{-b \pm \sqrt{b^{2}-4 a c}}{2 a}
$$

De la ecuación (2.c) se toman los coeficientes, $a=2 ; b=-4 ; c=-8$, para obtener:

$$
y=\frac{-b \pm \sqrt{b^{2}-4 a c}}{2 a}=\frac{-(-4) \pm \sqrt{(-4) 2-4(2)(-8)}}{2(2)}
$$

Obsérvese que después de operar la expresión (2.e), se separan los dos términos del numerador con su respectivo denominador.

$$
\frac{4}{4} ; \frac{\sqrt{16+64}}{4}
$$

Entonces, la ecuación (2.e) queda de la siguiente manera:

$$
y=\frac{4 \pm \sqrt{16+64}}{4}=\frac{4}{4} \pm \frac{\sqrt{16+64}}{4}
$$

A continuación, elevamos el cuatro al cuadrado para ingresar este nuevo término a la raíz y de esta manera simplificar la ecuación.

$$
y=\frac{4}{4} \pm \sqrt{\frac{16}{16}+\frac{64}{16}}
$$

Se tiene

$$
y=1 \pm \sqrt{(1+4)}=1 \pm \sqrt{ } 5
$$

Es decir, los puntos de intersección son

$$
y_{1}=1+\sqrt{5} \quad y_{2}=1-\sqrt{5}
$$

Se redondea a cuatro cifras significativas $y_{1} \cong$ 3.236 y $y_{2} \cong-1.236$.

Luego, se toman los valores de $y_{1} y_{2^{\prime}}$ y se evalúan en la ecuación (2.a) o en la ecuación (2.b), siguiendo este proceso se obtendrá dos valores $x_{1}$ y $x_{2}$, estos dos valores corresponden a la imagen de $y_{1}$ y $y_{2}$ (la imagen es el valor de $x$ cuando el valor de $y$ se evalúa en la respectiva ecuación).

$$
\begin{gathered}
X_{1}=y^{2}-4 y \\
x(3.236)=(3.236)^{2}-4(3.236) \\
x(3.236)=-2.472 \\
x_{2}=y^{2}-4 y \\
x(-1.236)=(-1.236)^{2}-4(-1.236) \\
x(-1.236)=6.472
\end{gathered}
$$

Con los valores encontrados, el punto $\mathbf{p} 1$ se representa gráficamente como $(-2.472,3.236)$ y el punto p2, como $(6.472,-1.236)$. (Véase gráfica 2b) 


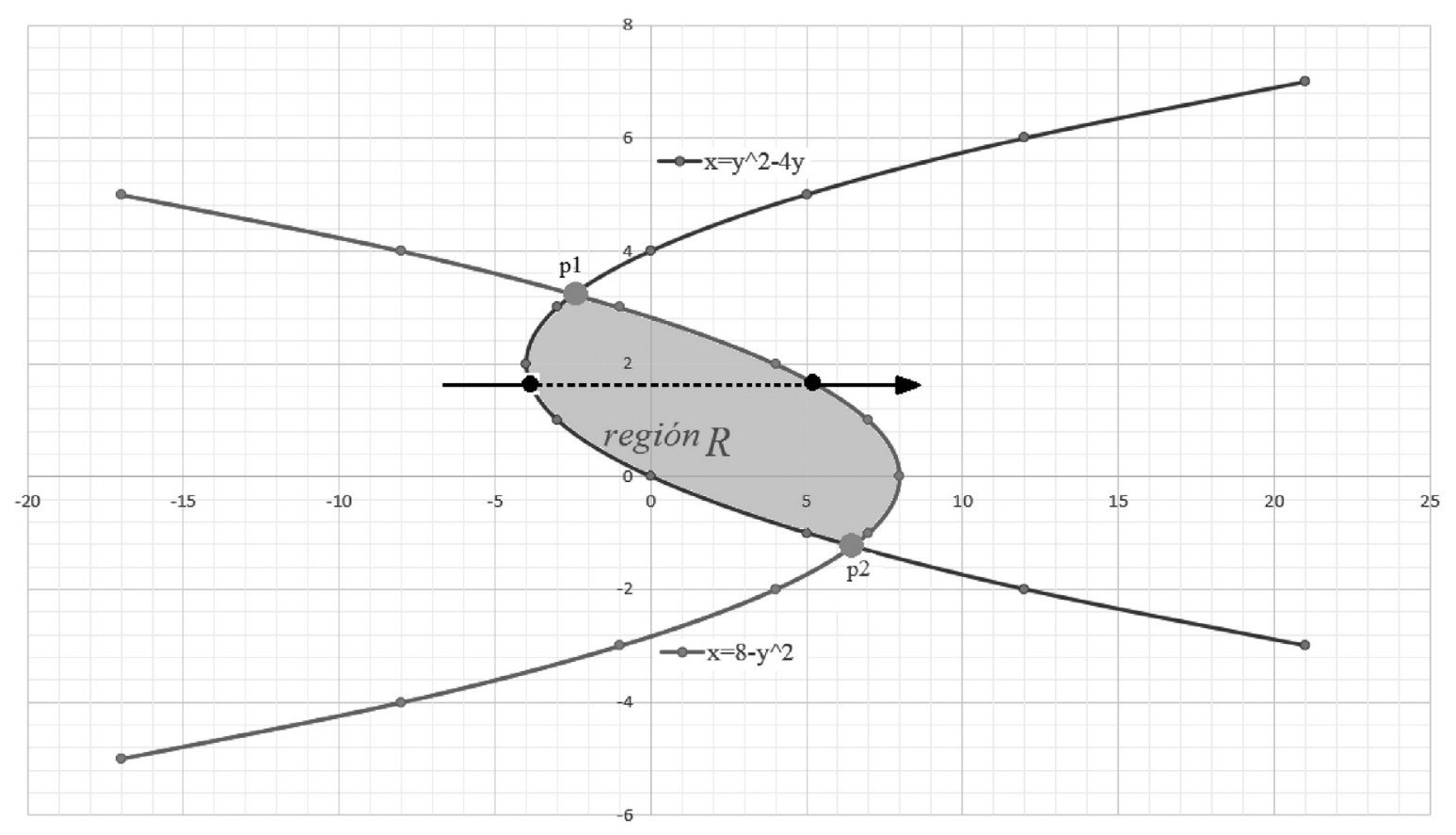

Gráfica 2b. Región $R$ y puntos $\mathrm{p} 1$ y p2

Una vez conocidos los puntos de intersección entre las dos ecuaciones, calculamos el área de la región $\mathrm{R}$ por medio de la integral doble $\iint_{R} d x d y$. Observe que la región $R$ es una región de tipo II ${ }^{4}$, por lo que el área se obtiene empleando una sola integral doble.

Para identificar los límites que acotan la pieza, se definen los límites de la integral doble, como sigue:

En primer lugar, se identifica el orden de integración. (dxdy o dydx)

4 Es de tipo II la región R que lleva como orden de integración dxdy, siendo los límites que definen la integral dx funciones únicamente con variable independiente y, y un número los límites que definen la integral dy. Obsérvese su definición matemática. (Salas, Hille, \& Etgen, 2002)

Tipo II: la proyección de $\boldsymbol{\Omega}$ en el eje $\boldsymbol{y}$ es un intervalo cerrado $[c, d]$ y $\boldsymbol{\Omega}$ consiste en todos los puntos $(x, y)$ tales que $\quad c \leq y \leq d \quad$ y $\quad \psi 1(\mathrm{y}) \leq x \leq$ $\psi 2(y)$ entonces $\iint_{\Omega} f(x, y) d x d y=\int_{c}^{d}\left(\int_{\psi 1(y)}^{\psi 2(y)} f(x, y) d x\right) d y$

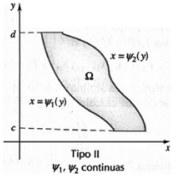

La integral doble que se utilizará es $\iint_{R}=d x d y$. Identificar el orden de las variables de integración es relevante para definir de manera correcta los límites de integración; primero se integra respecto a la variable $x y$ luego respecto a la variable $y$.

En segundo lugar, se definen los límites de la integral respecto a $d x$ :

La línea punteada de la Gráfica $\mathbf{2 b}$, paralela al eje $x$, corta las dos parábolas horizontales de izquierda a derecha. Estos puntos de intersección corresponden a los límites de la integral, de tal forma que el valor de entrada a la región $\boldsymbol{R}$ es $x=y^{2}-4 y$, que corresponde al límite inferior de la integral, y el valor de salida de la región $\boldsymbol{R}$ es $x=8-y^{2}$, que corresponde al límite superior de la integral.

Límites de la integral respecto a $d y$ :

Los límites del área $R$ con respecto a la coordenada y corresponden a los puntos de intersección P1 y P2 de la Gráfica 2b. Es decir, el 
límite inferior es $y=-1.236$ y el límite superior es $y=3.236$.

Por lo tanto el área de la figura plana se obtiene solucionando la siguiente integral:

$$
A=\int_{-1.236}^{3.236} \int_{y 2-4 y}^{8-y 2} d x d y
$$

Se resuelve la integral respecto a dx y luego se evalúa entre los límites de integración

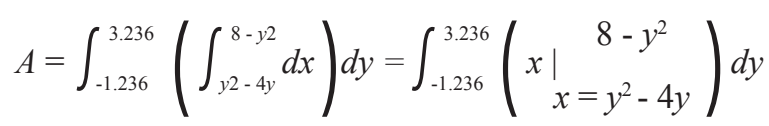

Luego de evaluar el límite superior e inferior, se simplifica la ecuación obtenida.

$$
A=\int_{-1.236}^{3.236}\left(8-y^{2}-\left(y^{2}-4 y\right)\right) d y=\int_{-1.236}^{3.236}\left(8-2 y^{2}+4 y\right) d y
$$

Como se va a integrar respecto a la variable $y$, se debe tener en cuenta que la función a integrar no contenga ningún factor dependiente de $x$.

Con el fin de facilitar la resolución de la integral respecto a dy se aplica la regla de la suma. ${ }^{5}$

$$
A=\int_{-1.236}^{3.236} 8 d y-\int_{-1.236}^{3.236} 2 y^{2} d y+\int_{-1.236}^{3.236} 4 y d y
$$

Se evalúa respecto a y

$$
A=\left.8 y\right|_{y=-1.236} ^{3.236}-\frac{2 \mathrm{y}^{3}}{3}\left|\begin{array}{c}
3.236 \\
y=-1.236
\end{array}+\frac{4 \mathrm{y}^{2}}{2}\right|_{y=-1.236}^{3.236}
$$

$\mathrm{A}=8(3.236)-8(-1.236)-\frac{2(3.236)^{3}}{3}+\frac{2(-1.236)^{3}}{3}+2(3.236)^{2}-2(-1.236)^{2}$

5 Regla de la suma: la integral definida de una suma (o resta) de funciones integrables es igual a la suma (o resta) de sus integrales definidas.
Se opera

$\mathrm{A} \cong 25.888+9.888-22.59-1.259+20.94-3.055$

$$
\mathrm{A} \cong 29.81 \mathrm{u}^{2}
$$

El área de la figura plana es aproximadamente $29.81 u^{2}$.

- Cuando se desea calcular el área R con el orden de integración inverso, es decir $\mathrm{A}=\iint_{R} d y d x$, es necesario conocer las ecuaciones de las curvas en función de la variable $x$ y además identificar los límites de integración, los que se determinaran a continuación.

Inicialmente se tenían las ecuaciones $x=y^{2}$ $4 y$ y $x=8-y^{2}$ que corresponden a las parábolas horizontales de la Gráfica 2b.

Para reescribir las dos ecuaciones anteriores en función de $x$ tenemos que

$$
x=y^{2}-4 y
$$

Se pasan todos los términos a la izquierda y se iguala a 0

$$
y^{2}-4 y-x=0
$$

Observe que la anterior ecuación es una parábola. Para conseguir que x sea la variable independiente se hace uso de la función cuadrática

$$
y=\frac{-(-4) \pm \sqrt{(-4)^{2}-4(1)(-x)}}{2(1)}
$$


Se realizan las operaciones hasta conseguir simplificar la expresión

$$
\begin{gathered}
y=\frac{4 \pm \sqrt{16+4 x}}{2}=\frac{4}{2} \pm \sqrt{\frac{16}{4}+\frac{4 x}{4}} \\
y=2 \pm \sqrt{4+x}
\end{gathered}
$$

Para $x=8-y^{2}$ se tiene que

$$
y^{2}=8-x
$$

Aplicando raíz cuadrada a ambos lados de la igualdad se obtiene

$$
y= \pm \sqrt{8-x}
$$

De la ecuación (2.f) y (2.g) se obtienen las siguientes ecuaciones

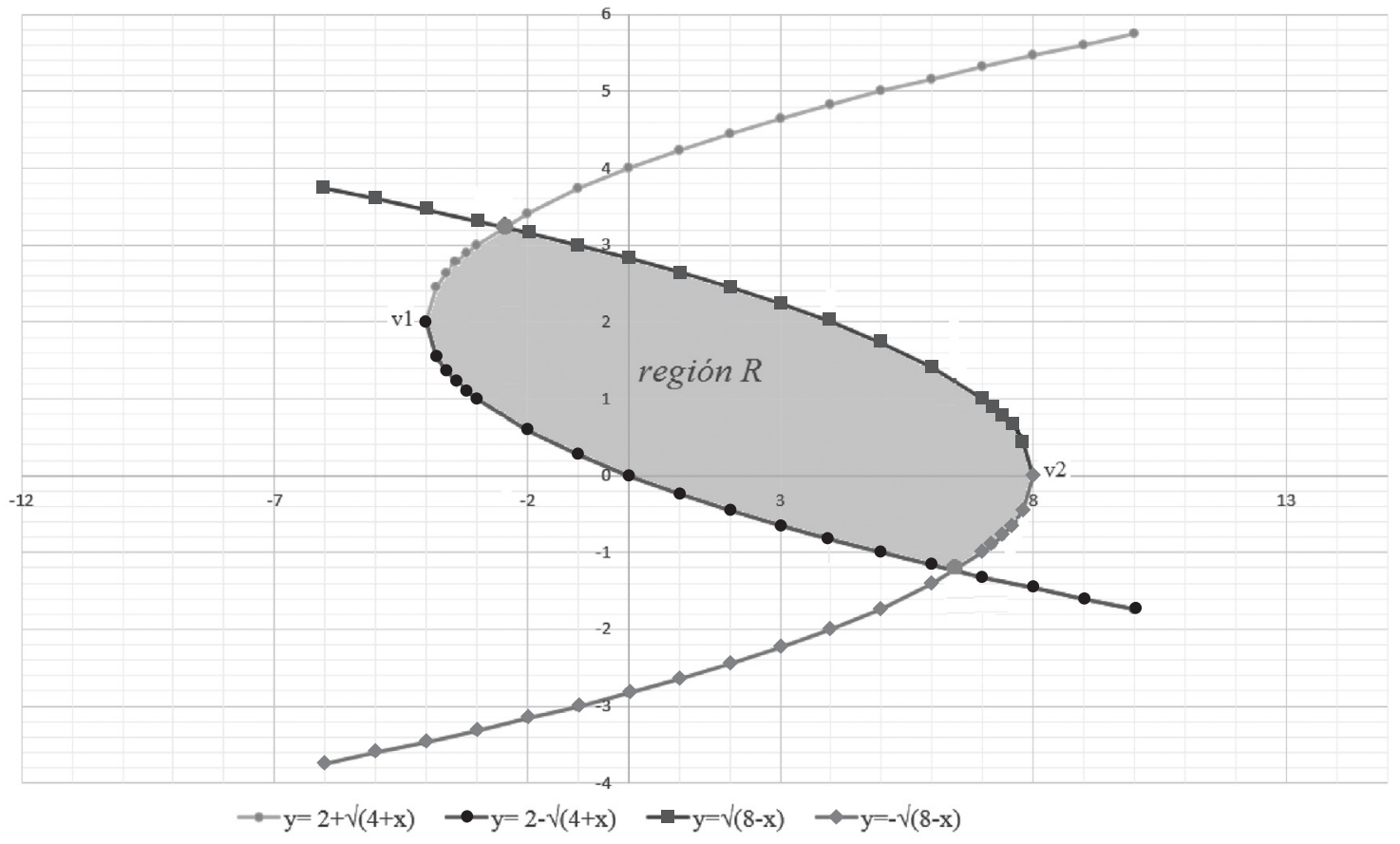

Gráfica 2c. $y 1=2+\sqrt{ }(4+x), y 2=2-\sqrt{ }(4+x)$,

$$
y 3=\sqrt{ }(8-x), y 4=-\sqrt{ }(8-x)
$$

Las ecuaciones anteriores corresponden a los límites de integración respecto a dy (véase Gráfica 2c). Tanto el vértice de cada parábola (v1 y v2) como los puntos de intersecion en la coordenada $x$ hacen parte de los límites de integración respecto a $d x$.
A continuación se hallará el vértice de las ecuaciones: $x=y^{2}-4 y$ y $x=8-y^{2}$

Se identifican los valores de $a, b$ y $c$ de la primera ecuación $x=y^{2}-4 y$ siendo $a=1, b=-4$ yc $=0$, para la segunda ecuación $x=8-y^{2}$ donde $a=-1, b=0$ y $c=8$. 
Luego, se utiliza la fórmula del vértice (2.h) para hallar el valor de la variable independiente, en este caso el valor de $y .{ }^{6}$

$$
y_{v}=\frac{-b}{2 a}
$$

Para la primera ecuación

$$
y_{v 1}=\frac{-(-4)}{2(1)}=\frac{4}{2}=2
$$

Enseguida, se sustituye $y_{v 1}$ en la primera ecuación por su valor numérico para hallar el valor de $x_{v 1}$. Es posible pensar en la fórmula para hallar el vértice de una función cuadrática de la siguiente forma: $(x, y)=\left(\frac{-b}{2 a}, f\left(\frac{-b}{2 a}\right)\right)$. Es decir que para calcular el valor de $x_{v 1}$ es necesario despejar la incógnita $\mathrm{y}_{\mathrm{v} 1}$ utilizando la fórmula, para después evaluar el valor numérico hallado en la ecuación.

$$
\mathrm{x}_{\mathrm{v} 1}=f \frac{-b}{2 a}=(2)^{2}-4(2)=4-8=-4
$$

El vértice de la primera ecuación es

$$
v_{1}=\left(\frac{-b}{2 a}, f\left(\frac{-b}{2 a}\right)\right)=(-4,2)
$$

Se realiza nuevamente el procedimiento anterior para obtener el vértice de la segunda ecuación

$$
v_{2}=\left(\frac{-b}{2 a}, f\left(\frac{-b}{2 a}\right)\right)=(8,0)
$$

Después de obtener los vértices v1 y v2, se trazan dos líneas verticales que cortan los puntos de intersección p1 y p2 con el fin de garantizar que las nuevas regiones únicamente estén acotadas por dos funciones

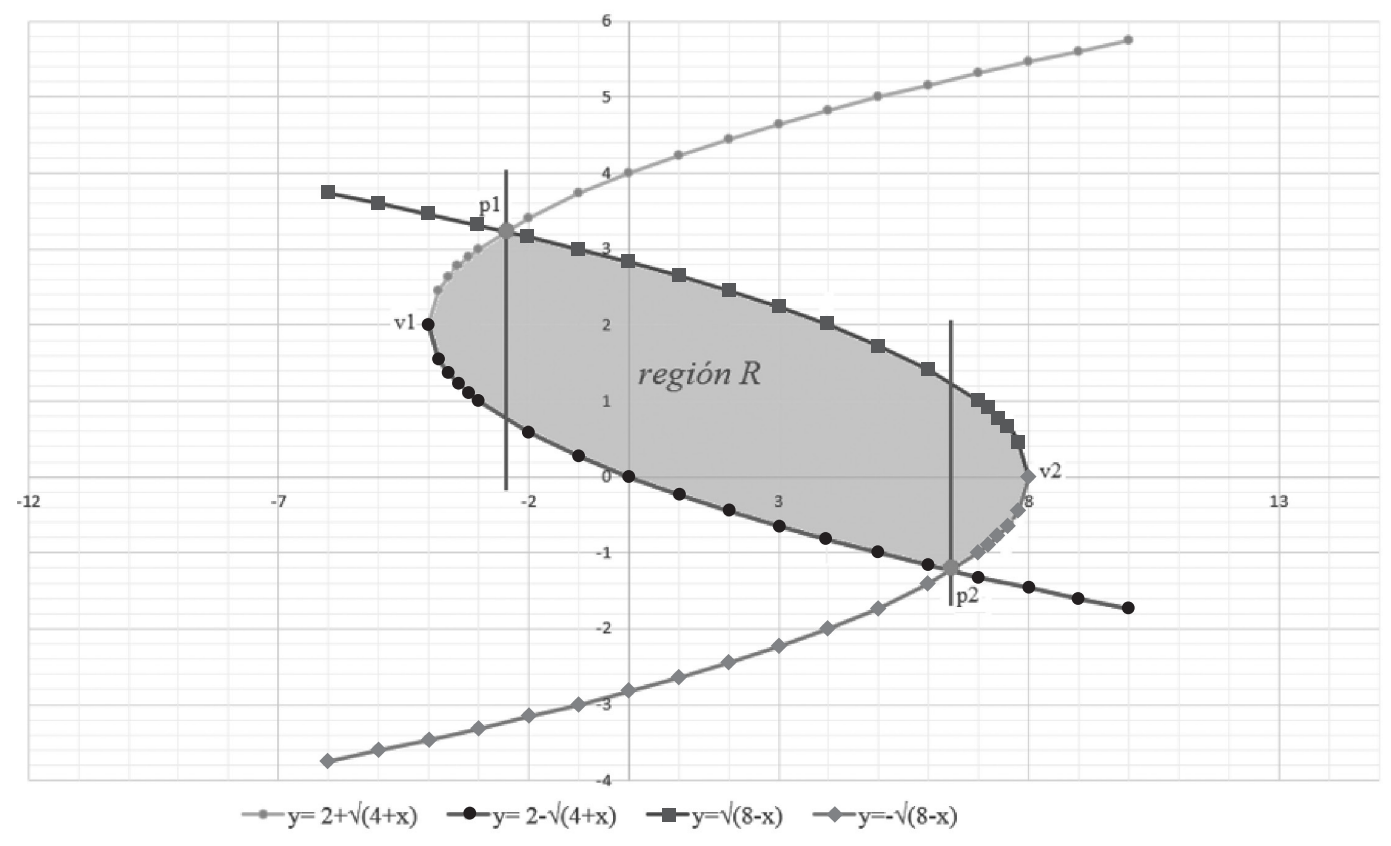

Gráfica 2d. Puntos de intersección p1 y p2

6 El vértice también determina dónde se encuentra el eje de simetría de la ecuación. 
Las dos líneas dividen la región $R$ en tres regiones $R 1, R 2$ y $R 3$ (véase Gráfica 2e.)

Se traza una flecha vertical (color negro) de abajo hacia arriba por cada región, el punto de entrada y el punto de salida cortarán una única función (véase Gráfica 2f).

Para hallar el área total (región $R$ ) de la gráfica anterior se necesita plantear tres integrales tipo $I^{7}$, identificadas como $R 1, R 2$ y $R 3$.

$$
\begin{aligned}
& R I=\int_{-4}^{-2.472} \int_{2-\sqrt{4+x}}^{2+\sqrt{4+x}} d y d x \\
& R 2=\int_{-2.472}^{6.472} \int_{2-\sqrt{4+x}}^{\sqrt{8-x}} d y d x \\
& R 3=\int_{6.472}^{8} \int_{-\sqrt{8-x}}^{\sqrt{8-x}} d y d x
\end{aligned}
$$

Obsérvese que los límites respecto a $d x$ son los valores numéricos que limitan en el eje $x$ las regiones $R 1, R 2$ y $R 3$.

Se inicia resolviendo la integral $R 1$ respecto a $d y$, para luego ser evaluada entre los límites de integración. Los siguientes pasos los aplicamos también a $R 2$ y $R 3$.

$R I=\int_{-4}^{-2.472} \int_{2-\sqrt{4+x}}^{2+\sqrt{4+x}} d y d x=\int_{-4}^{-2.472}\left(\begin{array}{c}\left.y\right|_{y=2-\sqrt{4+x}} ^{2+\sqrt{4+x}} \\ y=2\end{array}\right) d x$

Se evalúan los límites en el resultado de la integral respecto a $d y$ (límite superior menos límite inferior) y se opera la expresión matemática.

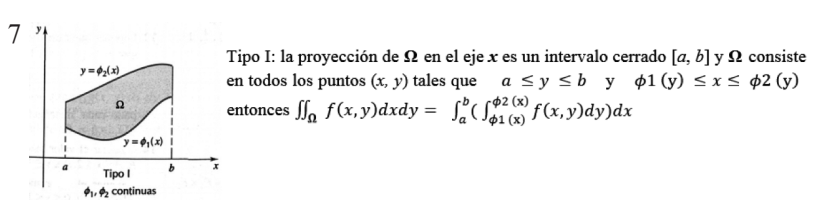

$$
\begin{gathered}
\int_{-4}^{-2.472}(2+\sqrt{4+x}-(2-\sqrt{4+x})) d x= \\
\int_{-4}^{-2.472}(2+\sqrt{4+x}-2+\sqrt{4+x}) d x
\end{gathered}
$$

Después de evaluar y operar la expresión se observa que queda un nuevo integrando únicamente en función de $x$, lo que permite integrar respecto a $d x$ sin ningún problema.

Se escogió el siguiente método porque es práctico. No obstante, el método que se implementa a continuación hace uso de propiedades de la matemática fundamental.

Inicialmente, se separa el dos como constante de la función $\sqrt{(4+x)}$ a integrar.8

$$
\int_{-4}^{-2.472}(2 \sqrt{4+x}) d x=2 \int_{-4}^{-2.472} \sqrt{4+x} d x
$$

Se reescribe $\sqrt{(4+x)}$ como $(4+x)^{\frac{1}{2}}: 9$

$$
2 \int_{-4}^{-2.472}(4+x)^{\frac{1}{2}} d x
$$

Luego, se consigue expresar en términos de una integral inmediata haciendo el siguiente cambio de variable. ${ }^{10}$

Derivamos ambos lados de la igualdad

$$
\begin{gathered}
u=4+x \\
d u=d x
\end{gathered}
$$

8 Propiedad de la integral: la integral del producto de una constante por una función es igual a la constante por la integral de la función.

9 Propiedad de los radicales: expresión de un radical en forma de potencia $\sqrt{a^{m}}=a^{\frac{m}{n}}$

10 Se conoce como una integral inmediata la integral de una potencia $\int f u^{n} * u^{\prime} d x=\frac{u^{n+1}}{n+1}+c \quad n \neq-1$ 


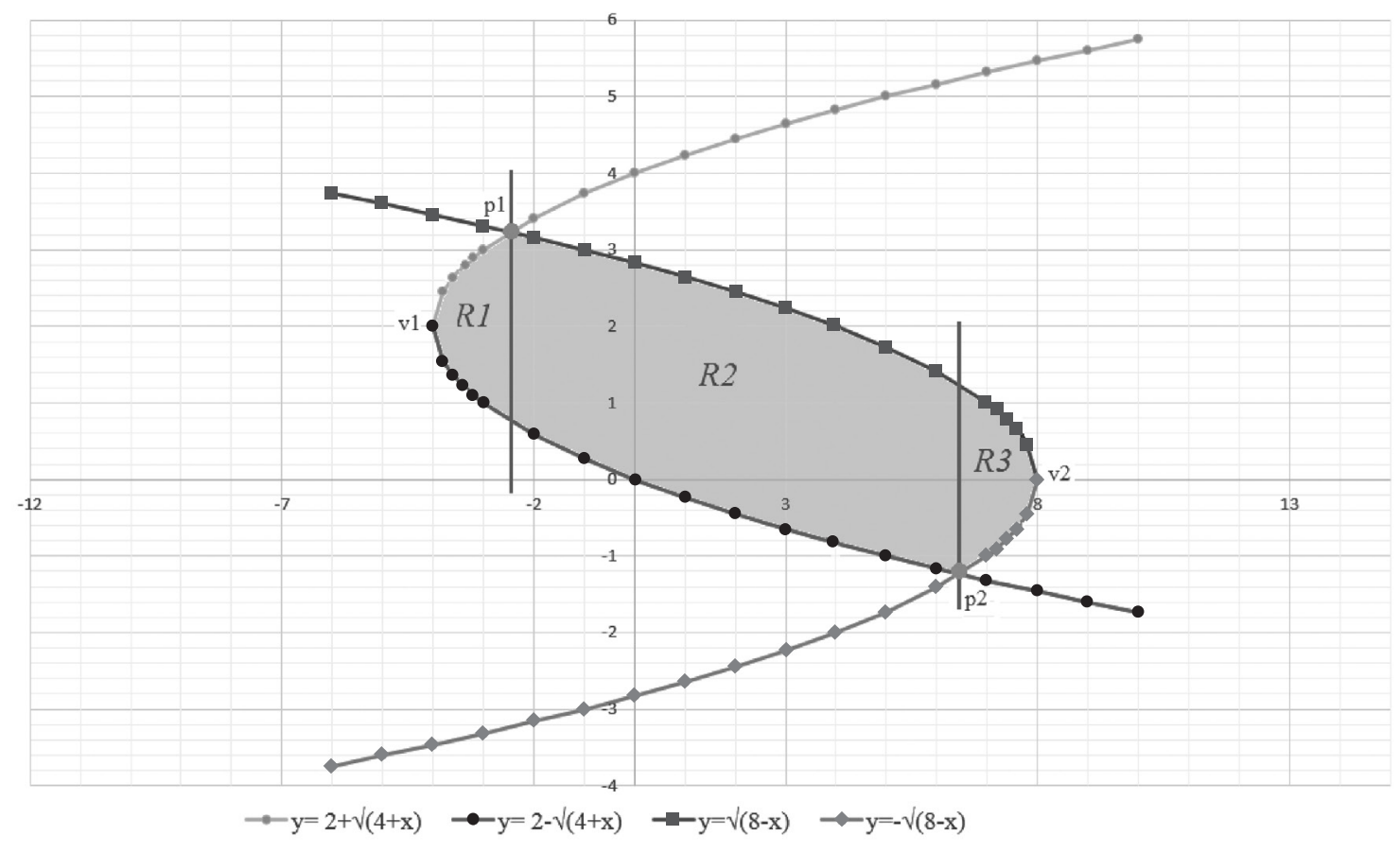

Gráfica 2e. Regiones $R 1, R 2$ y $R 3$

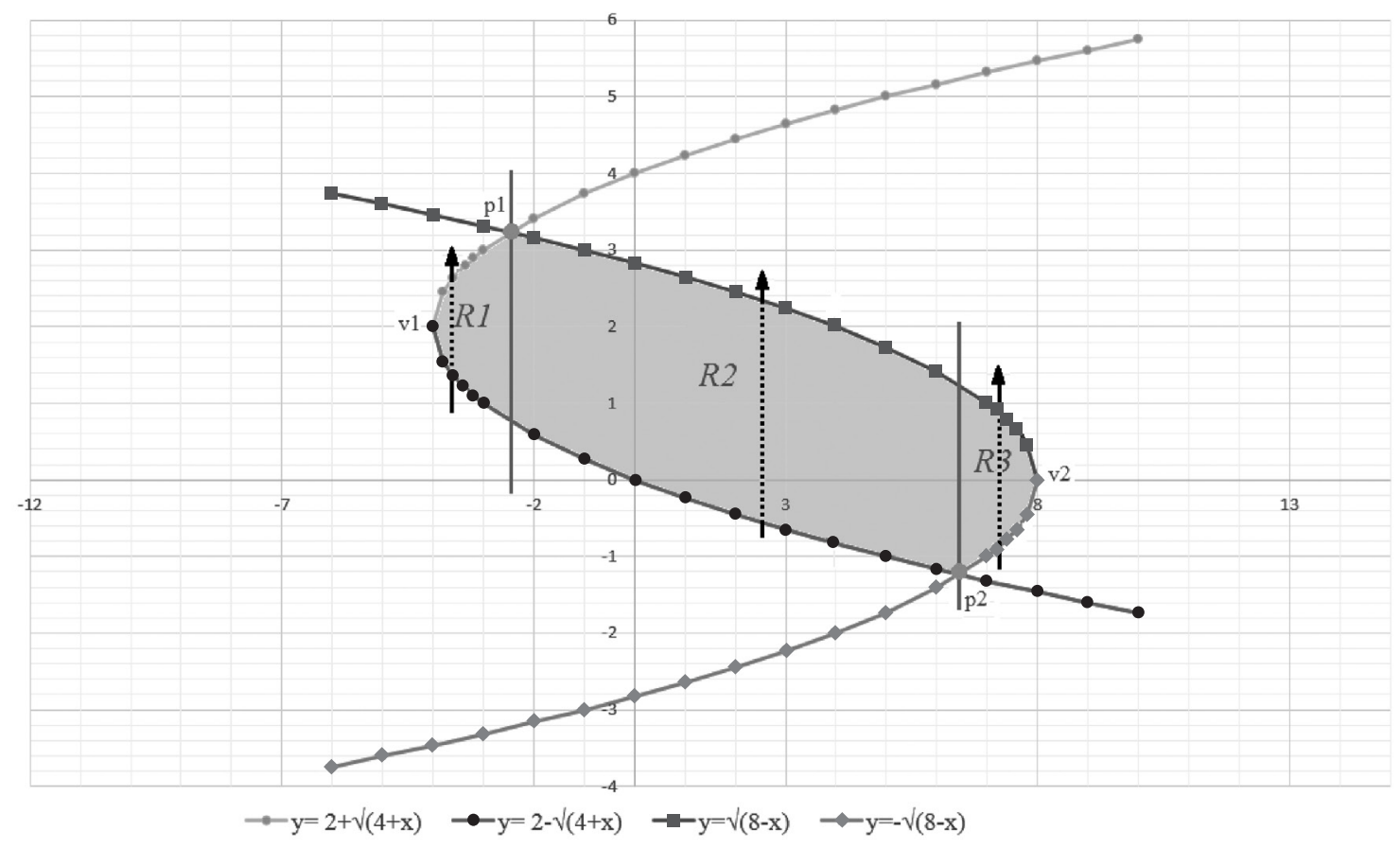

Gráfica 2f. Regiones $R 1, R 2$ y $R 3$ para las integrales de tipo I 
Obsérvese que la integral es definida y que se ha hecho cambio de variable, por tanto, es necesario calcular los nuevos límites de la integral, para ello se sustituye -2.472 en $x$ (límite superior) en la ecuación $\boldsymbol{u}=\mathbf{4}+\boldsymbol{x}$ y operamos. Este paso se repite para -4 (límite inferior)

$$
\begin{array}{cl}
u=4+(-2.472) & u=4+(-4) \\
u=1.528 & u=0
\end{array}
$$

Después de eso, se reescribe la función a integrar en términos del cambio de variable

$$
\begin{aligned}
& 2 \int_{0}^{1.528} u^{\frac{1}{2}} d u=\left.\frac{2 u^{\frac{3}{2}}}{\frac{3}{2}}\right|_{u=0} ^{1.528}=\frac{2(2) u^{\frac{3}{2}}}{3}=
\end{aligned}
$$

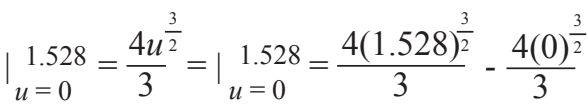

Cuando se resuelve la integral, se obtiene una división de dos fracciones $\frac{2 u^{\frac{3}{2}}}{\frac{3}{2}}$, la cual da otra fracción siendo el numerador de esta nueva fracción el producto de los extremos y el denominador el producto de los medios, de esta operación se obtiene como resultado $\frac{4 u^{\frac{3}{2}}}{3}$.

Enseguida, se evalúa el límite superior menos el límite inferior, lo cual da como resultado el área de la región $R 1$ que es aproximadamente $2.518 u^{2}$.

$$
R 1 \cong \frac{7.555}{3} \cong 2.518 u^{2}
$$

Para resolver la integral $R 2$ se procede primero a integrar respecto a $d y$

$$
R 2=\int_{-2.472}^{6.472} \int_{2-\sqrt{4+x}}^{\sqrt{8-x}} d y d x=\int_{-2.472}^{6.472}\left(\begin{array}{c}
\left.y\right|_{y=2-\sqrt{4+x}} ^{\sqrt{8-x}} \\
y=2
\end{array}\right) d x
$$

Se evalúa los límites y se opera la expresión a integrar

$$
\begin{gathered}
\int_{-2.472}^{6.472}(\sqrt{8-x}-(2-\sqrt{4+x})) d x= \\
\int_{-2.472}^{6.472}(\sqrt{8-x}-2+\sqrt{4+x}) d x
\end{gathered}
$$

Obsérvese que la integral respecto a $d x$ contiene tres funciones, lo cual permite aplicar la regla de la suma en el integrando para separar la integral anterior en tres integrales $R 2 a$, $R 2 b, R 2 c$.

$$
\int_{-2.472}^{6.472} \sqrt{8-x} d x+\int_{-2.472}^{6.472}-2 d x+\int_{-2.472}^{6.472} \sqrt{4+x} d x
$$

Se reescribe el radical en su forma de potencia

$$
R 2 a=\int_{-2.472}^{6.472} \sqrt{8-x} d x=\int_{-2.472}^{6.472}(8-\mathrm{x})^{\frac{1}{2}} d x
$$

Igual que con la integral $R 1$ se hace uso de un cambio de variable, siendo

$$
\begin{aligned}
& u=8-x \\
& d u=-d x \\
& -d u=d x
\end{aligned}
$$

Enseguida se calculan los nuevos límites de igual manera que con la integral $R 1$

$$
\begin{gathered}
u=8-(6.472) \\
u=1.528 \\
u=8-(-2.472) \\
u=10.47
\end{gathered}
$$

$$
\int_{10.47}^{1.528} u^{\frac{1}{2}}(-d u)=-\int_{10.47}^{1.528} u^{\frac{1}{2}} d u=\int_{1.528}^{10.47} u^{\frac{1}{2}} d u=\left.\frac{u^{\frac{3}{2}}}{\frac{3}{2}}\right|_{u=1.528} ^{10.47}
$$


En la anterior integral se observa que el límite superior es menor que el límite inferior y que además el $d u$ viene precedido por un signo negativo. Este caso llega a confundir al lector que idealiza el integrando gráficamente. Cuando se presenta la situación anterior, se puede invertir los límites conllevando a la eliminación del signo negativo. Es decir, la nueva integral definida será positiva ${ }^{11}$ con su límite superior mayor a su límite inferior.

$$
=\left.\frac{2 u^{\frac{3}{2}}}{3}\right|_{u=1.528} ^{10.47}=\frac{2(10.47)^{\frac{3}{2}}}{3}-\frac{2(1.528)^{\frac{3}{2}}}{3}
$$

Con el objetivo de hallar el área de la región $R 2 a$ se opera las fracciones obteniendo su mínima expresión.

$$
\cong \frac{67.76}{3}-\frac{3.778}{3} \cong 22.59-1.259 \cong 21.33 u^{2}
$$

La siguiente integral es inmediata, puesto que la funcion a integrar es una constante.

$R 2 b=\int_{-2.472}^{6.472}-2 d x=-2 \int_{-2.472}^{6.472} \mathrm{dx}=-\left.2 \mathrm{x}\right|_{x=-2.472} ^{6.472}=-2(6.472)+2(-2.472)$

Se integra respecto a $x$ para luego evaluar los respectivos límites $y$, por ende, obtener el área de la región $R 2 b$

$$
\cong-12.94-4.944 \cong-17.88 u^{2}
$$

La integral $R 2$ c es semejante a la integral $R 2 a$, lo que permite aplicar los mismos pasos de desarrollo de $R 2 a$ con $R 2 c$.

$$
R 2 c=\int_{-2.472}^{6.472} \sqrt{4+x} d x=\int_{-2.472}^{6.472}(4+\mathrm{x})^{\frac{1}{2}} d x
$$

11 Si $f$ es integrable en $[a, b]$ entonces $\int_{a}^{b} f(x) d x=-\int_{a}^{b} f(x) d x$
Se realiza un cambio de variable, donde

$$
\begin{aligned}
& u=4+x \\
& d u=d x
\end{aligned}
$$

Se calculan lo nuevos límites de igual manera que con la integral $R 2 a$

$$
\begin{aligned}
& u=4+(6.472) \\
& u=10.472 \\
& u=4+(-2.472) \\
& u=1.528 \\
& \int_{1.528}^{10.472} \frac{1}{u^{2}} d u=\left.\frac{u^{\frac{3}{2}}}{{ }^{\frac{3}{2}}}\right|_{u=1.528} ^{10.472}=\left.\frac{2 u^{\frac{3}{2}}}{3}\right|_{u=1.528} ^{10.472}=\frac{2(10.472)^{\frac{3}{2}}}{3}-\frac{2(1.528)^{\frac{3}{2}}}{3} \\
&=\frac{67.78}{3}-\frac{3.778}{3} \cong 22.59-1.259 \cong 21.33 u^{2}
\end{aligned}
$$

Nótese que el área de la región $R 2$ es aproximadamente la suma del área de las regiones $R 2 a, R 2 b$ y $R 2 c$, por tanto, el área de la región $R 2$ es equivalente a $24.78 u^{2}$.

$$
\begin{aligned}
& R 3=\int_{6.472}^{8} \int_{-\sqrt{8-x}}^{\sqrt{8-x}} d y d x=\int_{6.472}^{8}\left(\begin{array}{c}
y \mid \\
y=-\sqrt{8-x}
\end{array}\right) d x \\
& \int_{6.472}^{8}(\sqrt{8-x}-(-\sqrt{8-x})) d x=\int_{6.472}^{8}(\sqrt{8-x}+\sqrt{8-x}) d x \\
& \int_{6.472}^{8}\left(2 \sqrt{8-x} d x=\int_{6.472}^{8}(2 \sqrt{8-x})^{\frac{1}{2}} d x\right.
\end{aligned}
$$

Se realiza un cambio de variable, donde

$$
\begin{aligned}
& u=8-x \\
& d u=-d x \\
& -d u=d x
\end{aligned}
$$


Se calculan los nuevos límites de igual manera que con la integral $R 2 C$

$$
\begin{gathered}
u=8-(8) \\
u=0 \\
u=8-(6.472) \\
u=1.528 \\
\int_{1.528}^{0} 2 u^{\frac{1}{2}}(-d u)=-\int_{0}^{1.528} 2 u^{\frac{1}{2}} d u=\left.\frac{2 u^{\frac{3}{2}}}{\frac{3}{2}}\right|_{u=0} ^{1.528}=\left.\frac{2(2) u^{\frac{3}{2}}}{3}\right|_{u=0} ^{1.528} \\
=\left.\frac{4 u^{\frac{3}{2}}}{3}\right|_{u=0} ^{1.528}=\frac{4(1.528)^{\frac{3}{2}}}{3}-\frac{2(0)^{\frac{3}{2}}}{3} \cong \frac{7.555}{3} \cong 2.518 u^{2}
\end{gathered}
$$

El área de la región $R 3$ es aproximadamente $2.518 u^{2}$

Finalmente, el área de la región $R$ implementando el orden de integración $A=\iint_{R} d y d x$ es la suma del área de las tres regiones $R 1, R 2$ y $R 3$, lo cual da como resultado que el área de la figura plana es aproximadamente $29.81 u^{2}$.

Del ejercicio anterior se concluye que independientemente del orden de integración que se implemente $\left(A=\iint_{R} d x d y\right.$ o $\left.A=\iint_{R} d y d x\right)$, para determinar el área de una figura plana, el resultado es el mismo. Sin embargo, si se analiza el desarrollo de las dos integrales iteradas $\left(\iint_{R} d x d y\right.$ y $\left.\iint_{R} d y d x\right)$, la primera de tipo II y la segunda de tipo I, se observa que el desarrollo del integrando $\iint_{R} d y d x$ es más dispendioso que el desarrollo del integrando $\iint_{R} d x d y$, debido a que la región $R$ se convierte en tres regiones $R 1$, $R 2$ y $R 3$, lo cual aumenta el número de integrales a solucionar.

Se recomienda al lector que antes de iniciar con el desarrollo de cualquier ejercicio o situación que requiera hallar el área es necesario analizar qué tipo de integral iterada se va a utilizar, si es conveniente implementar un integrando de tipo I o de tipo II con el fin de facilitar el desarrollo de esta.
A continuación, se desarrollará un ejercicio que requiere hallar el área de una superficie plana implementando solamente un integrando que según el caso puede ser de tipo I o de tipo II.

\section{Cálculo del área de la superficie plana de una pieza en un sistema excéntrico}

Para ensamblar un sistema excéntrico se necesita diseñar una pieza en bronce, esta debe cumplir con unas medidas específicas: el ancho tomado del vértice $\mathbf{a}$ al vértice $\mathbf{b}$ debe ser de $7 \mathrm{u}$, la altura comprendida entre c y d es de 20 u y la profundidad 0.5 u (véase la Figura 4). Su proceso de fabricación exige conocer el área de la region $\mathrm{R}$.
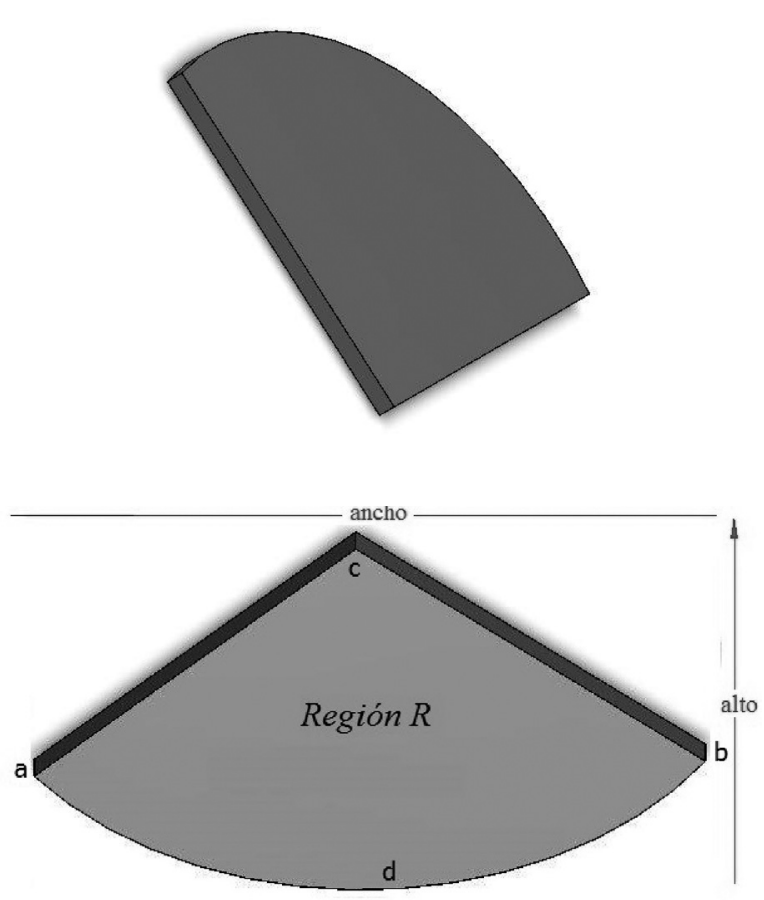

Figura 4. Pieza 2

Para hallar el área es indispensable conocer los límites que definen la integral doble, para ello se realizarán los siguientes pasos:

Cumpliendo con las condiciones mencionadas anteriormente, se parametrizará la pieza identificando la región $R$ (véase gráfica 3a). 


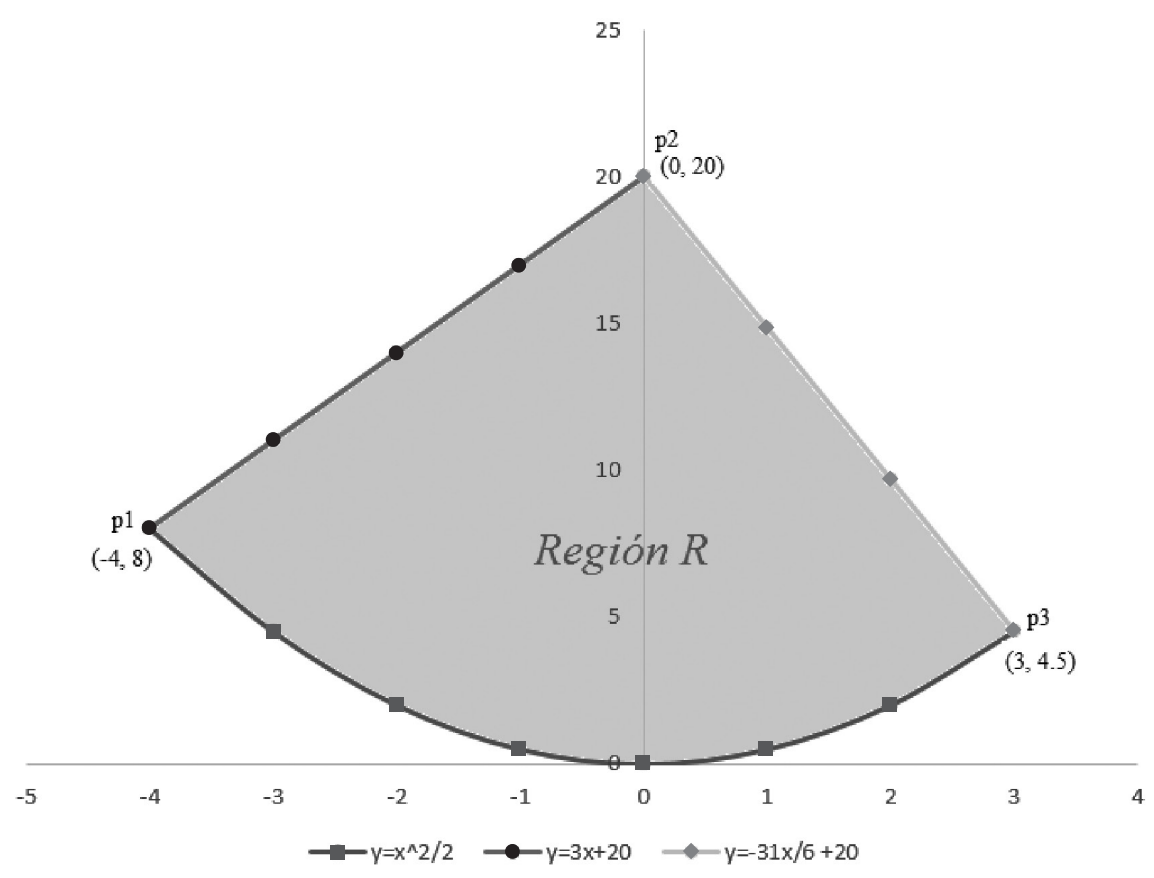

Gráfica 3a. Parametrización de la Pieza 2

Luego de parametrizar la pieza se determinará la ecuación de las curvas que limitan la región $R$. Se recurrirá a la ecuación de la recta y el método de interpolación parabólica, métodos que se desarrollarán a continuación.

Para el cálculo de la ecuación de la recta p1 p2 se utilizarán los puntos $\mathrm{p} 1=(-4,8)$ y p2 $=(0,20)$.

El primer paso es calcular la pendiente $\left(m_{1}\right)^{12}$.

$$
\begin{gathered}
m_{1}=\frac{y_{2}-y_{1}}{x_{2}-x_{1}} \\
m_{1}=\frac{20-8}{0-(-4)}=\frac{12}{4}=3
\end{gathered}
$$

El segundo paso consiste en sustituir la pendiente y cualquiera de los dos puntos $\mathrm{p} 1$ o $\mathrm{p} 2$ en la ecuación de la recta, para este caso se

12 La pendiente $\left(m_{1}\right)$ es positiva, porque la recta es creciente. escoge el punto p2. No obstante, si se escogiera el punto $\mathrm{p} 1$ la ecuación hallada sería la misma.

$$
\begin{gathered}
y-y_{2}=m_{1}\left(x-x_{2}\right) \quad \text { ecuación de la recta } \\
y-20=3(x-0) \\
y=3 x+20
\end{gathered}
$$

Se utilizarán los puntos $\mathrm{p} 2=(0,20)$ y $\mathrm{p} 3=(3$, 4.5) para el cálculo de la ecuación de la recta p2p3.

Cálculo pendiente $\left(m_{2}\right)^{13}$.

$$
m_{2}=\frac{y_{2}-y_{1}}{x_{2}-x_{1}}
$$

$$
m_{2}=\frac{4.5-20}{3-0}=\frac{-15.5}{3}=\frac{-15.5(2)}{3(2)}=\frac{-31}{6}
$$

13 La pendiente $\left(m_{2}\right)$ es negativa, porque la recta decrece. 
Para este caso se escoge el punto p2

$$
\begin{gathered}
y-y_{2}=m_{2}\left(x-x_{2}\right) \quad \text { ecuación de la recta } \\
y-20=\frac{-31}{6}(x-0) \\
y=\frac{-31}{6} x+20
\end{gathered}
$$

Un método para determinar la ecuación de una parábola es la interpolación parabólica que consiste en tomar tres puntos por los cuales pasa la función, estos se sustituyen en la ecuación canónica de la parábola, obteniendo un sistema de tres ecuaciones con tres incógnitas.

Puntos pertenecientes a la parábola (véase Gráfica 3.a).

$$
(-4,8) \quad(0,0) \quad(3,4.5)
$$

Ecuación canónica de la parábola

$$
y=a x^{2}+b x+c
$$

Para el primer punto $(-4,8)$

$$
\begin{gathered}
y=a x^{2}+b x+c \\
8=a(-4)^{2}+b(-4)+c \\
8=16 a-4 b+c \\
16 a-4 b+c=8
\end{gathered}
$$

Para el segundo punto $(0,0)$

$$
\begin{aligned}
& y=a x^{2}+b x+c \\
& 0=a(0)^{2}+b(0)+c \\
& 0=c \\
& c=0
\end{aligned}
$$

Para el tercer punto $(3,4.5)$

$$
\begin{aligned}
y & =a x^{2}+b x+c \\
4.5 & =a(3)^{2}+b(3)+c \\
4.5 & =9 a+3 b+c \\
9 a+3 b+c & =4.5
\end{aligned}
$$

Con la ecuación (3.a), (3.b) y (3.c) se plantea un sistema de ecuaciones de 3 por 3

$$
\left\{\begin{array}{r}
16 a-4 b+c=8 \\
c=0 \\
9 a+3 b+c=4.5
\end{array}\right.
$$

Obsérvese que se conoce el valor de $c=0$ esto permite reducir el sistema de tres ecuaciones con tres incógnitas a un sistema de dos ecuaciones con dos incógnitas de la siguiente manera:

Inicialmente, se sustituye el valor de c en la ecuación (3.a) y (3.c)

$$
\left\{\begin{array}{l}
16 a-4 b+(0)=8 \\
9 a+3 b+(0)=4.5
\end{array}=\left\{\begin{array}{l}
16 a-4 b=8 \\
9 a+3 b=4.5
\end{array}\right.\right.
$$

Luego se despeja $b$ de la ecuación (3.e). No obstante, si el lector lo desea puede despejar $a$ y el resultado obtenido será el mismo

$$
b=\frac{4.5}{3}-\frac{9 a}{3}
$$

Se sustituye la ecuación (3.f) en la ecuación (3.d) para hallar el valor de $a$

$$
16 a-4\left(\frac{4.5}{3}-\frac{9 a}{3}\right)=8
$$




$$
\begin{gathered}
16 a-\frac{18}{3}+\frac{36 a}{3}=8 \\
16 a+\frac{36 a}{3}=8+\frac{18}{3} \\
a\left(16+\frac{36}{3}\right)=14 \\
a=\frac{14}{28}=\frac{1}{2}
\end{gathered}
$$

Conociendo el valor de $a$ se sustituye en la ecuación (3.d) o (3.e) para determinar que $b=0$.
Después de conocer el valor del coeficiente $a, b$ y c la ecuación de la parábola es:

$$
y=\frac{1}{2} x^{2}
$$

Después de haber hallado las tres ecuaciones que limitan la región $R$, se analiza el orden de integración a utilizar, minimizando el número de integrales a solucionar.

Si se escogiera el orden de integración $\iint_{R} d x d y$, sería necesario dividir la región $R$ en tres regiones $R 1, R 2$ y $R 3$. Nótese que la región $R$ no es una región tipo II, sin embargo, las tres regiones si lo serían (véase Gráfica 3.b).

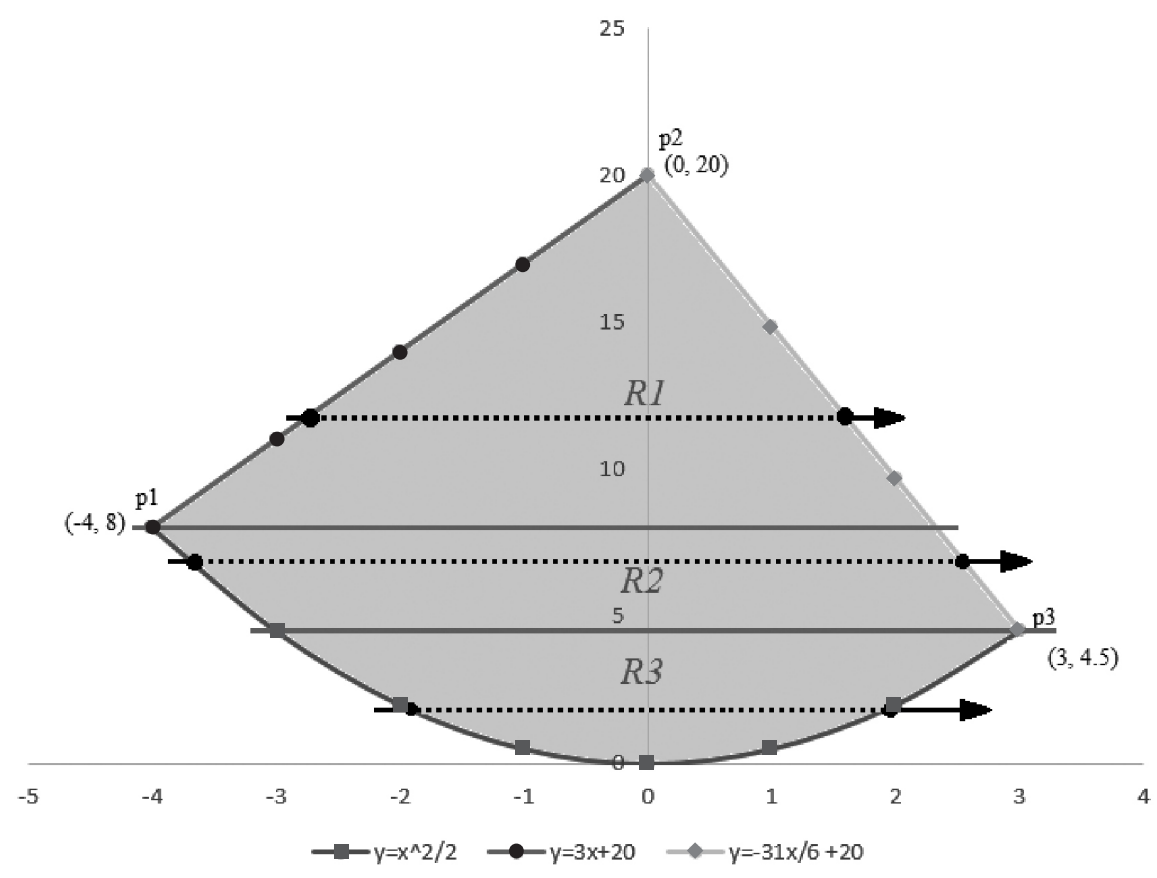

Gráfica 3.b. Orden de integración $\iint_{\mathrm{R}} d x d y$

Obsérvese que las ecuaciones que describen los límites del área están en función de la variable $x$, por lo que es necesario reescribir cada uno en función de y para determinar los límites de los integrandos.
Nótese, (Gráfica 3.b) que el valor de $x$ a la entrada de la región $R 1$ es la ecuación $x=\frac{y-20}{3}$ $y$ el valor de $x$ a la salida de la región $R 1$ es la ecuación $x=\frac{-6 y+120}{31}$. 
La región $R 1$ está en y acotada entre 8 y 20 .

El valor de $x$ a la entrada de la región $R 2$ es la ecuación $x=-\sqrt{2 y}$ y el valor de $x$ a la salida de la región $R 2$ es la ecuación $x=\frac{-6 y+120}{31}$ (véase Gráfica 3.b).

La región $R 2$ está en y acotada entre 4.5 y 8 .

El valor de $x$ a la entrada de la región $R 2$ es la ecuación $x=-\sqrt{2 y}$ y el valor de $x$ a la salida es la ecuaciónh $x=\sqrt{2 y}$. La región $R 3$ está en $y$ acotada entre 0 y 4.5 (véase Gráfica 3.b).
Después del análisis anterior las integrales que describen el área de la región $R$ son
Si se escogiera el orden de integración $\iint_{R}{ }_{R} d y d x$, sería necesario dividir la región $R$ en dos regiones de tipo I identificadas como $R 1$ y R2 (véase Gráfica 3.c).

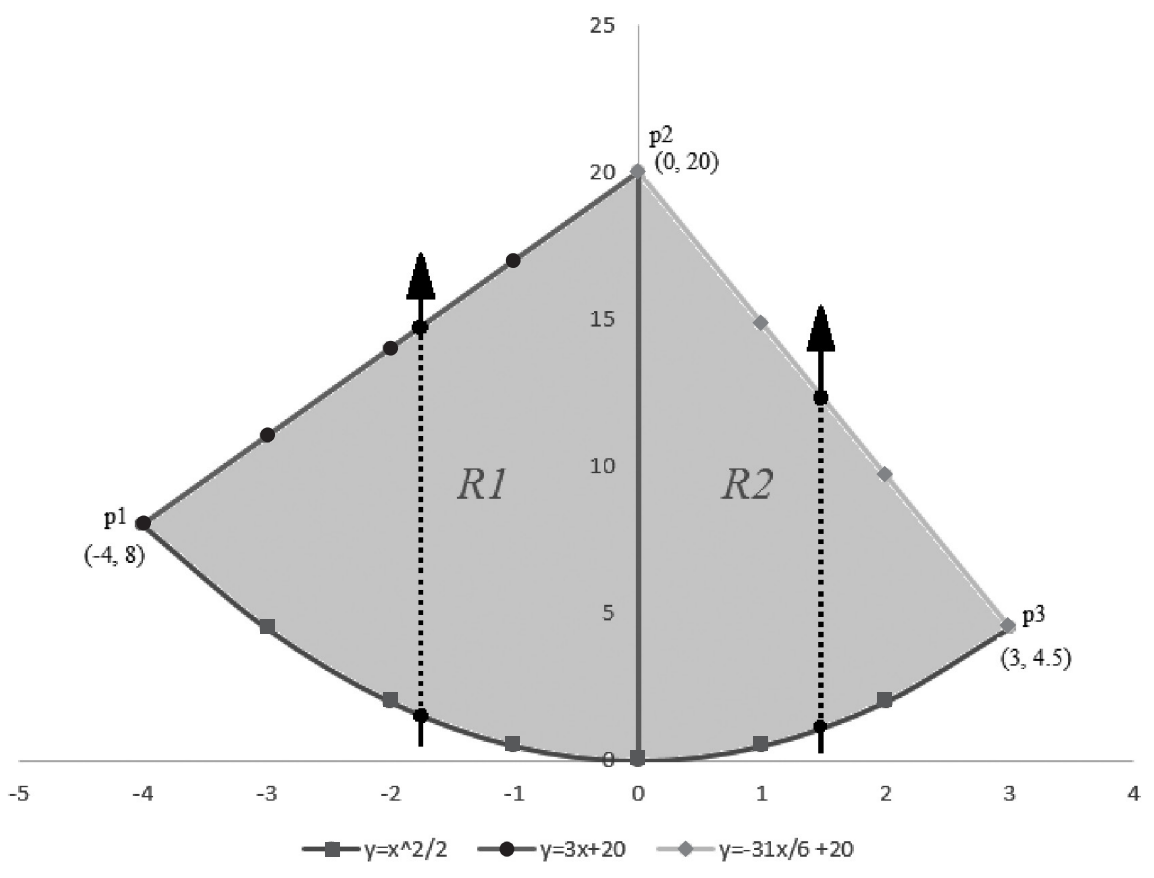

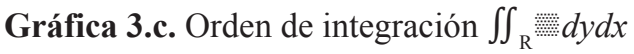

Obsérvese que el valor de y a la entrada de $R 1$ es $y=\frac{1}{2} x^{2}$ y que el valor de $y$ a la salida es $y=3 x$ +20 . Para $R 2$ el valor de $y$ a la entrada es $y=\frac{1}{2} x^{2}$ $y$ el valor de $y$ a la salida es $y=\frac{-31 x}{6}+20$.

La región $R 1$ está acotada en $x$ entre -4 y 0 , la región $R 2$ está acotada en $x$ entre 0 y 3 (véase Gráfica 3.c).
Dado el análisis anterior, las integrales que describen el área de la región $R$ son

$$
A=\int_{-4}^{0} \int_{\frac{1}{2} x^{2}}^{3 x+20} d y d x+\int_{0}^{3} \int_{\frac{1}{2} x^{2}}^{\frac{-31 x}{6}+20} d y d x
$$

Después de analizar los dos casos se escoge el segundo, puesto que solo habrá que resolver dos integrales para obtener el área de la 
superficie plana y no tres integrales como en el primer caso. Sin embargo, el lector puede desarrollar cualquiera de las dos posibilidades obteniendo al final la misma respuesta.

Solución primera integral

$$
\begin{gathered}
\int_{-4}^{0} \int_{\frac{1}{2} x^{2}}^{3 x+20} d y d x=\int_{-4}^{0}\left(\left.y\right|_{y=\frac{1}{2} x^{2}} ^{3 x+20}\right) d x=\int_{-4}^{0}\left(3 x+20-\frac{1}{2} x^{2}\right) d x= \\
\int_{-4}^{0} 3 x d x+\int_{-4}^{0} 20 d x-\int_{-4}^{0} \frac{x^{2}}{2} d x= \\
\frac{3 x^{2}}{2}|x \underline{0}-4+20 x| x \underline{0}-4-\frac{x^{3}}{6} x^{\underline{0}}-4= \\
\frac{3(0)^{2}}{2}-\frac{3(-4)^{2}}{2}+20(0)-20(-4)-\frac{(0)^{3}}{6}+\frac{(-4)^{3}}{6}= \\
-\frac{3(-4)^{2}}{2}-20(-4)+\frac{(-4)^{3}}{6}= \\
-\frac{48}{2}+80-\frac{64}{6}=\frac{136}{3} u^{2}
\end{gathered}
$$

Solución segunda integral

$$
\begin{aligned}
& \int_{0}^{3} \int_{\frac{1}{2} x^{2}}^{\frac{-33 x}{6}+20} d y d x=\int_{0}^{3}\left(\left.y\right|_{y=\frac{1}{2} x^{2}} ^{\frac{-31 x}{6}+20}\right) d x=\int_{0}^{3}\left(\frac{-31 x}{6}+20-\left(\frac{1}{2} x^{2}\right)\right) d x= \\
& \int_{0}^{3} \frac{-31 x}{6} d x+\int_{0}^{3} 20 d x-\int_{0}^{3} \frac{x^{2}}{2} d x= \\
& \frac{-31 x^{2}}{12}|x \underline{\underline{\underline{3}}} 0+20 x| x \underline{\underline{3}} 0-\frac{x^{3}}{6} \mid x \underline{\underline{3}} 0 \\
& -\frac{31(3)^{2}}{12}-\frac{31(0)^{2}}{12}+20(3)-20(0)-\frac{(3)^{3}}{6}+\frac{(0)^{3}}{6}= \\
& -\frac{31(3)^{2}}{12}-20(3)+\frac{(3)^{3}}{6}= \\
& -\frac{93}{4}+60-\frac{27}{6}=\frac{129}{4} u^{2}
\end{aligned}
$$

El área de la superficie plana es

$$
\frac{136}{3}+\frac{129}{4}=\frac{931}{12} u^{2} \cong 77.58 u^{2}
$$

Cálculo del volumen de un helado en un instante de tiempo

Un día en el parque, un joven quiso hallar el volumen del helado que estaba comiendo en ese instante, para ello obtuvo experimentalmente las siguientes ecuaciones de las superficies que acotan el volumen del sólido $z=3 \sqrt{x^{2}+y^{2}} y z=\sqrt{4-x^{2}-y^{2}}$.

Cuando se pedía hallar el área de una superficie plana, inicialmente se parametrizaban y graficaban las funciones que encerraban la región en el plano $x y$. No era indispensable tener nociones avanzadas de graficación, puesto que se utilizaba el método de tabulación. El caso contrario sucede cuando necesitamos hallar el volumen, ya que se requiere conocer las ecuaciones canónicas de la superficie de las figuras geométricas con el objetivo de poder idealizar a priori el sólido y establecer relaciones con volúmenes muy comunes. ${ }^{14}$

Es relevante identificar y relacionar las ecuaciones del enunciado con las ecuaciones canónicas para hacer un bosquejo del sólido. Enseguida se detallará, paso a paso, la solución del problema.

$$
\begin{aligned}
& z=3 \sqrt{x^{2}+y^{2}} \\
& z=\sqrt{4-x^{2}-y^{2}}
\end{aligned}
$$

Elevamos al cuadrado cada igualdad, para eliminar los radicales

$$
\begin{gathered}
z^{2}=\left(3 \sqrt{x^{2}+y^{2}}\right)^{2} \\
z^{2}=\left(\sqrt{4-x^{2}-y^{2}}\right)^{2}
\end{gathered}
$$

Eliminados los radicales operamos cada expresión

$$
z^{2}=9\left(x^{2}+y^{2}\right)
$$

14 En el apéndice están las ecuaciones canónicas fundamentales de las figuras geométricas tridimensionales. 


$$
\begin{gathered}
z^{2}=9 x^{2}+9 y^{2} \\
z^{2}=4-x^{2}-y^{2} \\
x^{2}+y^{2}+z^{2}=4
\end{gathered}
$$

Nótese que la ecuación (4.c) describe una superficie cónica y la ecuación (4.d) describe una esfera centrada en el punto $(0,0,0)$ de radio 2 .
Como se puede apreciar en la Gráfica 4a, la superficie inferior del sólido es igual a un cono descrito por la ecuación (4.a). Basado en la ecuación (4.d) correspondiente a una esfera, se determina que la ecuación (4.b) corresponde a una semiesfera debido al radical que contiene la expresión matemática (véase Gráfica 4b).

Ahora se sabe que el sólido está formado por la intersección (línea anaranjada) de un cono y una semiesfera como se muestra a continuación:

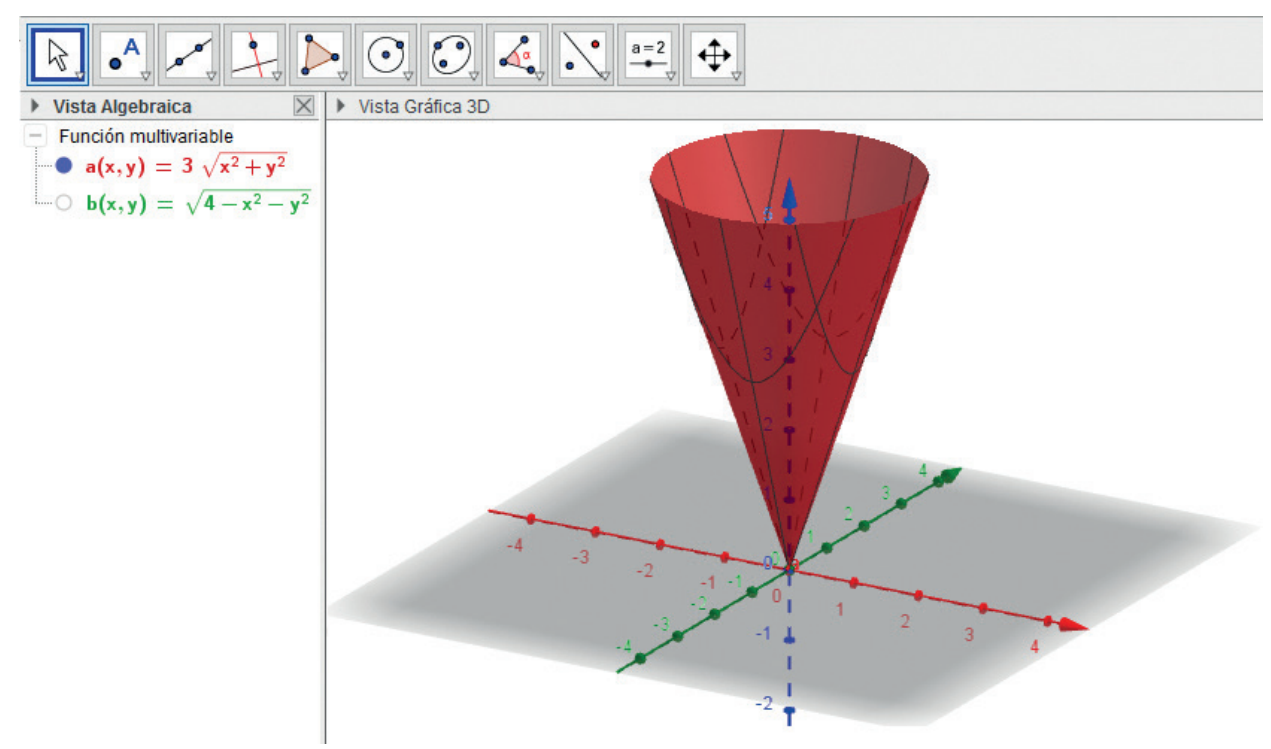

Gráfica 4a. Superficie cónica

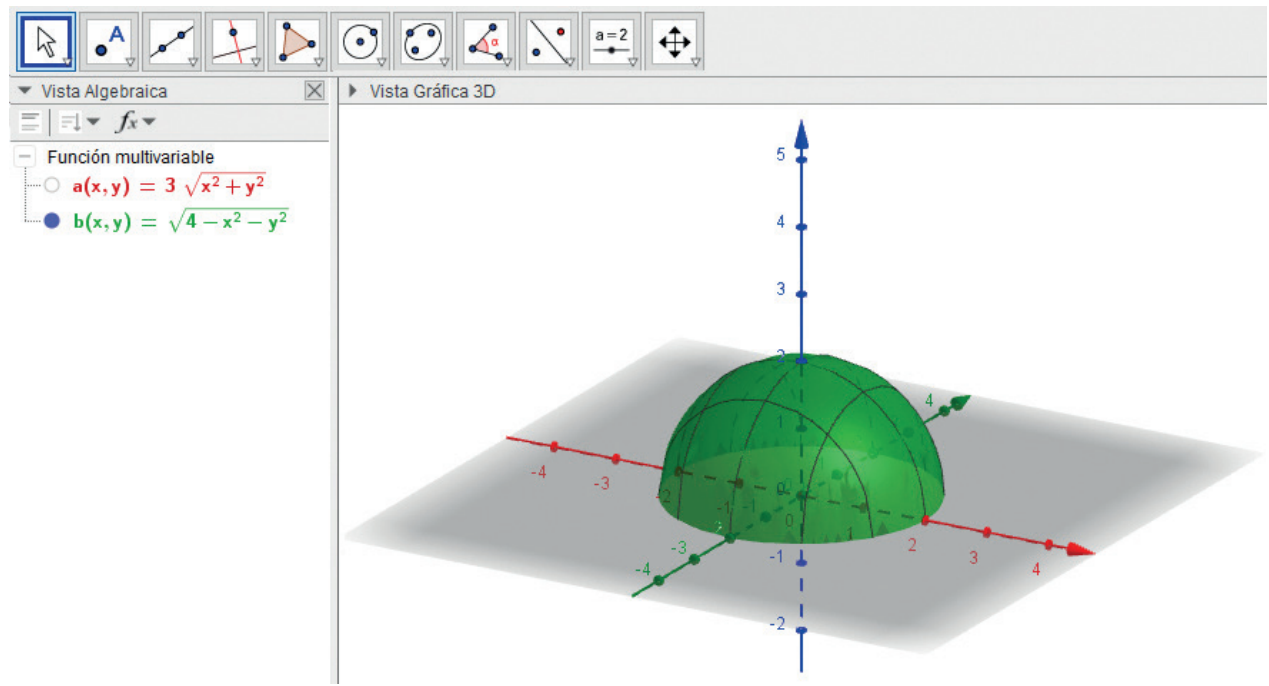

Gráfica 4b. Esfera centrada en el punto $(0,0,0)$ 


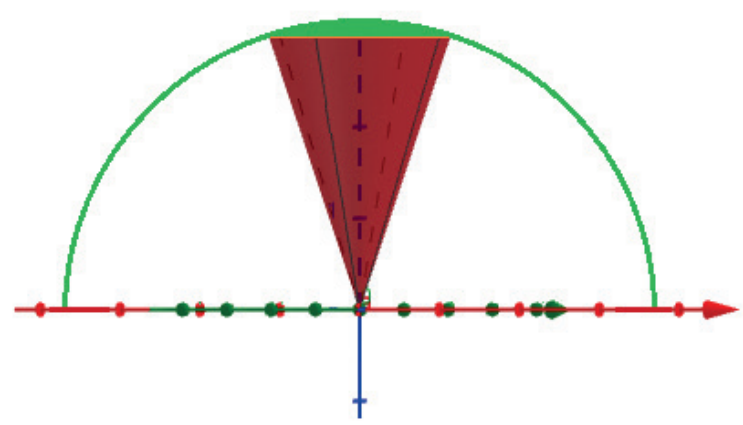

Gráfica 4c. Intersección entre la semiesfera (color verde) y el cono (color marrón).

El volumen de una región se define como la diferencia de dos funciones una superior y otra inferior que acotan el sólido.

Se tiene que:

$$
\begin{aligned}
v & =\iint_{\mathrm{R}}^{=} f(x, y) d A=\iint_{\mathrm{R}}^{=}[f(x, y)-h(x, y)] d A \\
& =\iint_{\mathrm{R}}^{=}[f(x, y)-h(x, y)] d x d y
\end{aligned}
$$

Basado en el concepto anterior, se determinan las dos funciones del integrando trazando una flecha de la parte inferior a la parte superior del sólido

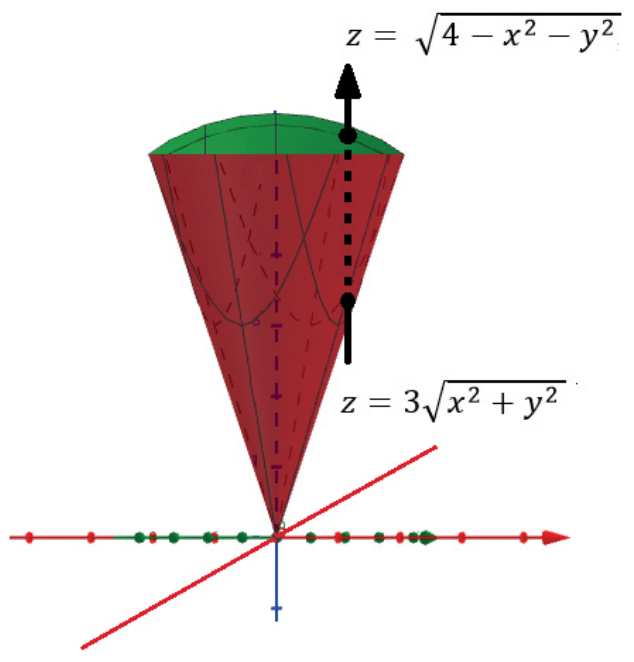

Gráfica 4d. La superficie definida por la ecuación $z=\sqrt{4-x^{2}-y^{2}}$ es una semiesfera (parte superior) y la superficie definida por la ecuación $z=3 \sqrt{x^{2}+y^{2}}$ es un cono.
Obsérvese que el valor de $z$ a la entrada del sólido es igual a $z=3 \sqrt{x^{2}+y^{2}}$ y el valor de $z$ a la salida del sólido es igual a $z=\sqrt{4-x^{2}-y^{2}}$, siendo la función de salida $f(x, y)$ y la función de entrada $h(x, y)$. Reemplazando en la integral (4.e) se obtiene:

$$
v=\iint_{\mathrm{R}}\left[\sqrt{4-x^{2}-y^{2}}-3 \sqrt{x^{2}+y^{2}}\right] d A
$$

Es necesario conocer la ecuación que describe la intersección entre el cono y la semiesfera para hallar los límites de la integral.

A continuación, se igualan las ecuaciones (4.a) y (4.b) para obtener la ecuación que intercepta al cono con la semiesfera.

$$
\begin{aligned}
3 \sqrt{x^{2}+y^{2}} & =\sqrt{4-x^{2}-y^{2}} \\
\left(3 \sqrt{x^{2}+y^{2}}\right)^{2} & =\left(\sqrt{4-x^{2}-y^{2}}\right)^{2} \\
9\left(x^{2}+y^{2}\right) & =\left(4-x^{2}-y^{2}\right) \\
10\left(x^{2}+y^{2}\right) & =4 \\
x^{2}+y^{2} & =\frac{2}{5}
\end{aligned}
$$

La ecuación (4.g) es el círculo que se denota con la línea de intercepción de color anaranjado entre el cono y la esfera que se visualiza en la gráfica 4c.

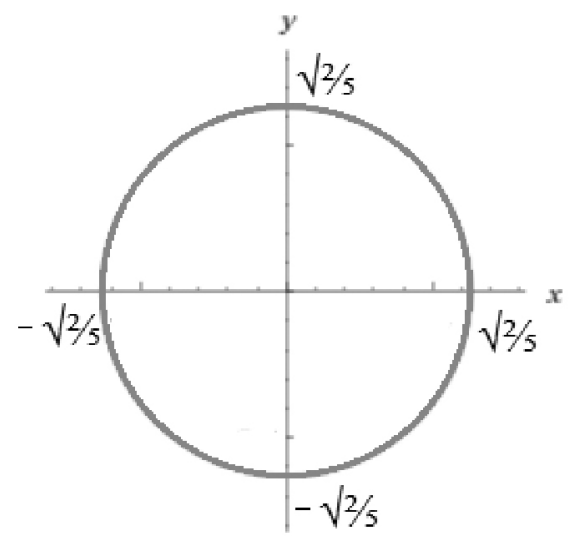

Gráfica 4e. Intercepción dentre el cono y la esfera dada por la ecuación $x^{2}+y^{2}=\frac{2}{5}$ 
Definición de límites de la integral respecto a dy: Graficada la ecuación se traza una flecha vertical que corte el círculo

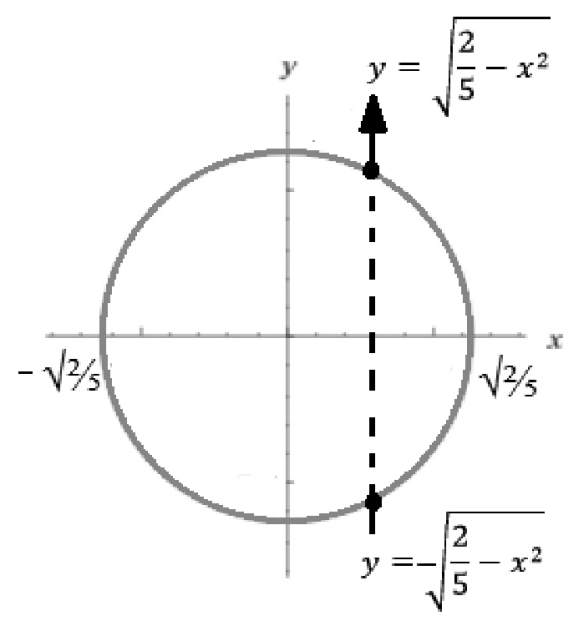

Gráfica 4f. Límites inferiores y superiores respecto a $d y$ y $d x$

Se toma como límite inferior el punto de entrada $y=-\sqrt{\frac{2}{5}-x^{2}}$ y como límite superior el punto de salida $y=\sqrt{\frac{2}{5}-x^{2}}$

Límites de la integral respecto a $d x$ :

Es el punto inicial y final del diámetro del círculo respecto al eje $\mathrm{x}$, siendo el límite inferior - $\sqrt{2 / 5}$ y el límite superior $\sqrt{{ }^{2 / 5}}$.

Nótese que el círculo está proyectado en el plano xy lo que conlleva a que los límites estén expresados únicamente en términos de dos variables $x$ y $y$.

Volviendo a la integral (4.f), se tiene que:

$$
v=\int_{-\sqrt{\frac{2}{5}}}^{\sqrt{\frac{2}{5}}} \int_{-\sqrt{\frac{2}{5}-x^{2}}}^{\sqrt{\frac{2}{5}-x^{2}}}\left[\sqrt{4-x^{2}-y^{2}}-3 \sqrt{x^{2}+y^{2}}\right] d y d x
$$

Para resolver esta integral se requiere un procedimiento muy riguroso y extenso, que se especificará enseguida.
Observe que la integral anterior no se puede resolver por ningún método común, por ende es necesario realizar un cambio de variable.

Se utilizarán las coordenadas polares expresadas como: ${ }^{15}$

$$
x=r \cos \theta \quad y=r \operatorname{sen} \theta
$$

Teniendo en cuenta lo anterior, la nueva integral se expresa como:

$$
\iint f(r \cos \theta, r \operatorname{sen} \theta) r d r d \theta^{16}
$$

Se sustituye a $x$ y a $y$ en las dos expresiones matemáticas de la integral (4.h) por su respectivo cambio de variable:

$$
\begin{aligned}
\sqrt{4-x^{2}-y^{2}} & =\sqrt{4-(r \cos \theta)^{2}-(r \operatorname{sen} \theta)^{2}} \\
& =\sqrt{4-r^{2} \cos ^{2} \theta-r^{2} \operatorname{sen}^{2} \theta} \\
& =\sqrt{4-r^{2}\left(\cos ^{2} \theta+\operatorname{sen}^{2} \theta\right)} \\
& =\sqrt{4-r^{2}} \\
3 \sqrt{x^{2}+y^{2}} & =3 \sqrt{(r \cos \theta)^{2}+(r \operatorname{sen} \theta)^{2}} \\
& =3 \sqrt{r^{2} \cos ^{2} \theta+r^{2} \operatorname{sen}^{2} \theta} \\
& =3 \sqrt{r^{2}\left(\cos ^{2} \theta+\operatorname{sen}^{2} \theta\right)} \\
& =3 \sqrt{r^{2}}=3 r
\end{aligned}
$$

Luego de sustituir el cambio de variable en las expresiones matemáticas, se determinan

15 En la parte final de la cartilla se enuncia el teorema de cambio a coordenadas polares en una integral doble.

$16(r)$ corresponde al jacobiano de las coordenadas polares. Se debe tener en cuenta que cada vez que se haga cambio de variable a coordenadas polares, cilíndricas o esféricas, la nueva integral debe tener su respectivo jacobiano. Véase en la parte final de la cartilla la demostración del jacobiano para coordenadas polares. 


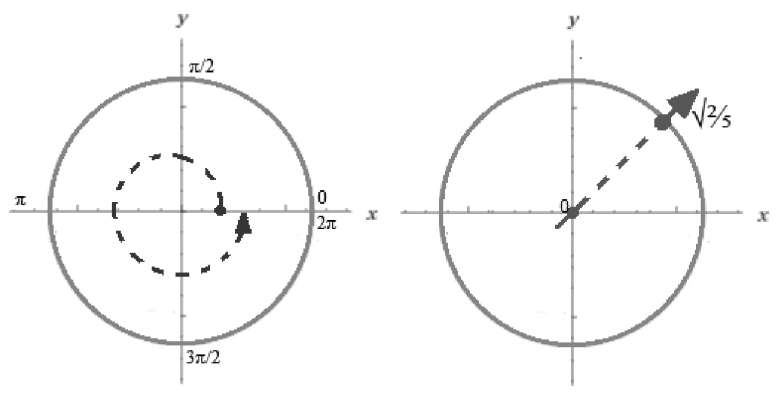

Gráfica 4g. Límites de la integral de acuerdo a las coordenadas polares

Para hallar los límites de $d \theta$ se traza una línea (color azul) que recorre toda el área, fijando un punto inicial en 0 (límite inferior) y un punto final en $2 \pi$ (límite superior). ${ }^{17}$

Los límites de $d r$ se obtienen trazando una flecha desde el centro del círculo (o proyección) hasta la línea anaranjada. Siendo el límite inferior igual a 0 y el límite superior igual a $\sqrt{2 / 5}$.

De esta manera la integral del volumen queda expresada como:

$$
\begin{gathered}
v=\int_{0}^{2 \pi} \int_{0}^{\sqrt{\frac{2}{5}}}\left[\sqrt{4-r^{2}}-3 r\right] r d r d \theta \\
v=\int_{0}^{2 \pi} \int_{0}^{\sqrt{\frac{2}{5}}}\left(r \sqrt{4-r^{2}}-3 r^{2}\right) d r d \theta=\int_{0}^{2 \pi} \int_{0}^{\frac{\sqrt{2}}{5}} r \sqrt{4-r^{2}} d r d \theta-\int_{0}^{2 \pi} \int_{0}^{\frac{\sqrt{2}}{5}} 3 r^{2} d r d \theta
\end{gathered}
$$

Se resuelve cada integral por aparte:

$$
\begin{aligned}
\int_{0}^{2 \pi} \int_{0}^{\sqrt{\frac{2}{5}}} r \sqrt{4-r^{2}} d r d \theta= & \\
u & =4-r^{2} \\
d u & =-2 r d r \\
\frac{-d u}{2} & =r d r
\end{aligned}
$$

Se calculan los nuevos límites:

$$
\begin{aligned}
& l_{i} \rightarrow u=4-(0)^{2}=4 \\
& l_{s} \rightarrow u=4-\left(\sqrt{\frac{2}{5}}\right)^{2}=\frac{18}{5}=3.6
\end{aligned}
$$

17 Se debe tener en cuenta que los límites que corresponden a $\theta$ siempre van a estar comprendidos entre 0 y $2 \pi$. No obstante, los límites respecto a $\theta$ se expresan en radianes.
Se reescribe en términos de $u$ :

$$
\begin{aligned}
\int_{0}^{2 \pi}\left(-\frac{1}{2} \int_{4}^{3.6} u^{\frac{1}{2}} d u\right) d \theta & =\int_{0}^{2 \pi}\left(\frac{1}{2} \int_{3.6}^{4} u^{\frac{1}{2}} d u\right) d \theta=\int_{0}^{2 \pi}\left(\left.\frac{u^{\frac{3}{2}}}{3}\right|_{u=3.6} ^{4}\right) d \theta \\
& =\int_{0}^{2 \pi}\left(\frac{(4)^{\frac{3}{2}}}{3}-\frac{(3.6)^{\frac{3}{2}}}{3}\right) d \theta=\left.\left(\frac{(4)^{\frac{3}{2}}}{3}-\frac{(3.6)^{\frac{3}{2}}}{3}\right) \theta\right|_{\theta=0} ^{2 \pi} \\
& =\left(\frac{(4)^{\frac{3}{2}}}{3}-\frac{(3.6)^{\frac{3}{2}}}{3}\right) 2 \pi
\end{aligned}
$$

$$
\begin{aligned}
-\int_{0}^{2 \pi} \int_{0}^{\sqrt{\frac{2}{5}}} 3 r^{2} d r d \theta=-\int_{0}^{2 \pi}\left(\frac{3 r^{3}}{3} I_{r=0}^{\sqrt{\frac{2}{5}}}\right) d \theta & =-\int_{0}^{2 \pi}\left(\left(\sqrt{\frac{2}{5}}\right)^{3}\right) d \theta=-\left.\left(\sqrt{\frac{2}{5}}\right)^{3} \theta\right|_{\theta=0} ^{2 \pi}= \\
& =-\left(\sqrt{\frac{2}{5}}\right)^{3} 2 \pi
\end{aligned}
$$

El volumen del helado es:

$$
\left(\frac{(4)^{\frac{3}{2}}}{3}-\frac{(3.6)^{\frac{3}{2}}}{3}\right) 2 \pi-\left(\sqrt{\frac{2}{5}}\right)^{3} 2 \pi
$$

Cálculo de la masa de una lámina de densidad no homogénea

La protección radiológica tiene por objeto la reducción de la dosis en las personas expuestas a la radiación. Para ello esta disciplina dispone de tres instrumentos bien conocidos: reducción del tiempo de exposición, aumento de la distancia a la fuente y blindaje.

La industria radiológica se encarga de estudiar diferentes propiedades de las láminas hechas por medio de aleaciones que poseen densidades no homogéneas, uno de estos estudios consiste en el cálculo de su masa para conseguir un excelente blindaje frente a las radiaciones electromagnéticas rayos $X$ y rayos Gamma.

El cálculo de la masa de una lámina es una de las interpretaciones más comunes de la integral doble. Enseguida, se explicará cómo determinar la masa de una figura plana no homogénea, de 
área $R^{18}$ para regiones donde la densidad varía en cada punto $(x, y) \in R .^{19}$

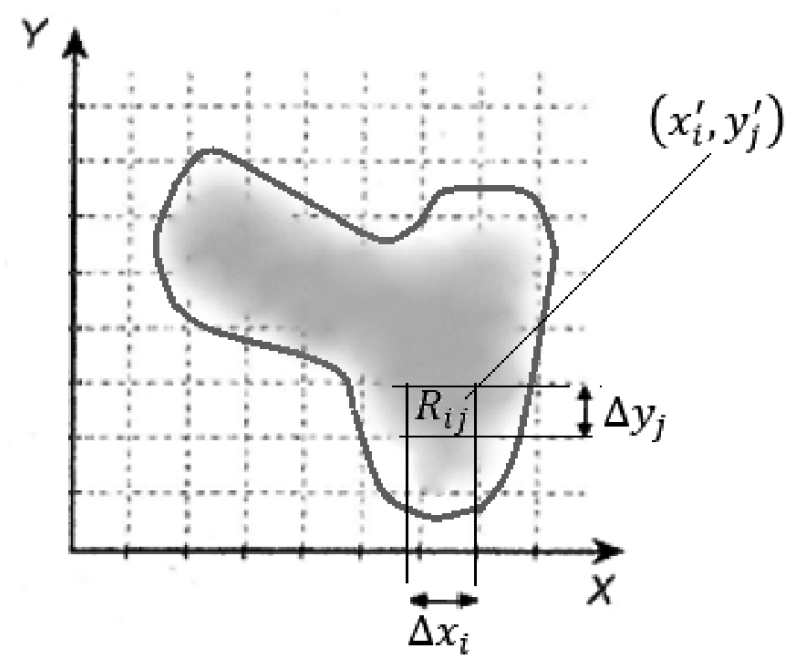

Figura 5a. La región $R$ no es homogénea, por lo cual su sombreado no es uniforme.

Se escoge un punto arbitrario $\left(x_{i}^{\prime} y_{j}^{\prime}\right) \in R_{i j}$ como se evidencia en la Figura $\mathbf{5 a}$, entonces la masa de este sub-rectángulo, denotada como $m_{\mathrm{ij}}$ se obtiene como:

$$
m_{i j}=\rho\left(x_{\mathrm{i}}^{\prime}, y_{\mathrm{j}}^{\prime}\right) \Delta A_{i j}
$$

Una aproximación de la masa de la placa plana de área $R$, se puede estimar mediante la doble suma de Riemann:

$$
m_{i j} \approx \sum_{j=1}^{n} \sum_{i=1}^{k} \rho\left(x_{i}^{\prime}, y_{j}^{\prime}\right) \Delta A_{i j}
$$

Para obtener la masa real de la placa se aumenta el número de sub-intervalos, de manera que la norma de la partición tienda a cero.

De este modo se obtiene la siguiente expresión en términos de sumatorias e integrales dobles:

$$
m=\lim _{\|P\| \rightarrow 0} \sum_{j=1}^{n} \sum_{i=1}^{k} \rho\left(x_{i}^{\prime}, y_{j}^{\prime}\right) \Delta A_{i j}=\iint_{R}^{\mathrm{m}} \rho(x, y) d A
$$

A continuación, se determina la masa de la placa plana Figura $\mathbf{5}$ b, cuya densidad ${ }^{20}$ varía de acuerdo a la función $\rho(x, y)=1+2 x$

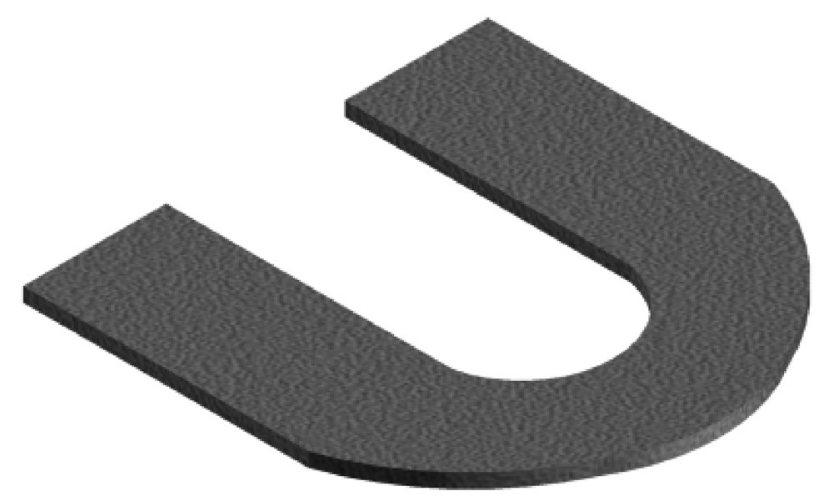

Figura 5b. Placa plana

Primero se parametriza la lámina con el fin de obtener las ecuaciones matemáticas que describen su forma.
18 Para esta aplicación se considera que la función densidad $\rho$ es continua en la región $R$.

19 Asimismo $\rho(x, y)=0$ para $(x, y)$ que no pertenezca a la región $R$.
20 La densidad tiene unidades de masa por área unitaria. 


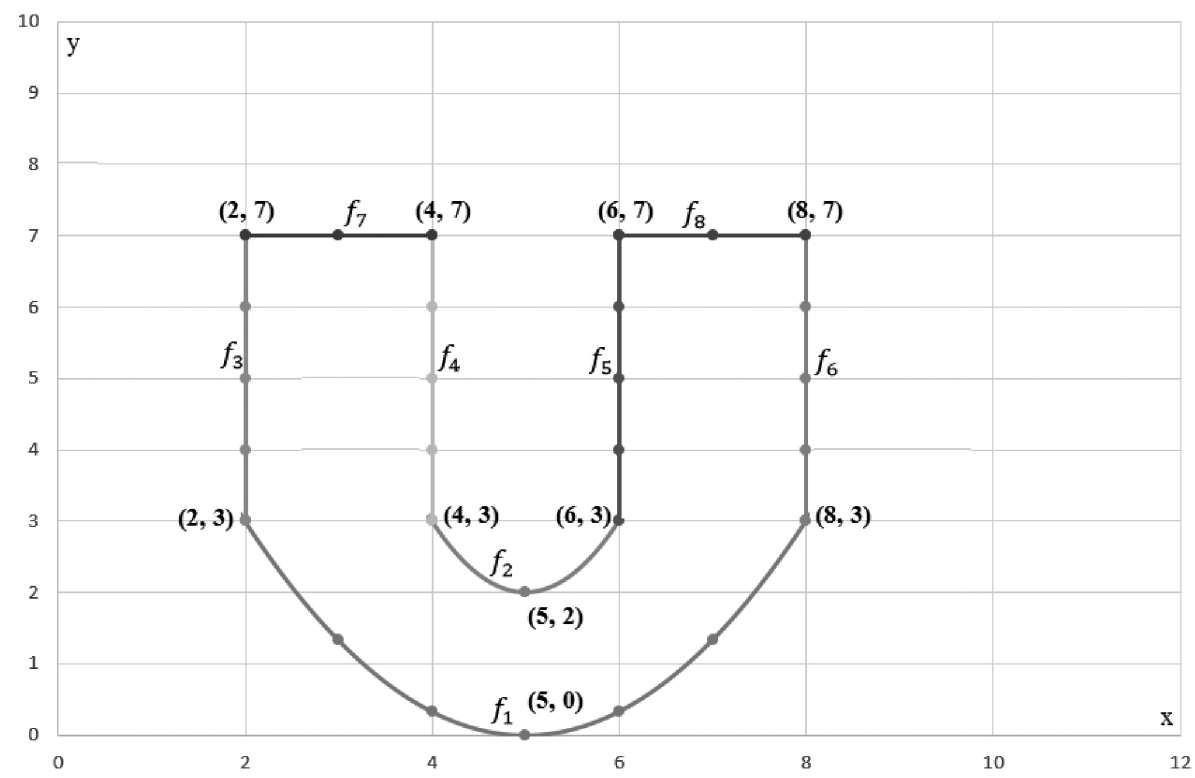

Gráfica 5.a. Forma de la placa

Para determinar la ecuación $f_{1}$ y $f_{2}$ se utiliza el método de interpolación parabólica como se muestra a continuación:

Puntos pertenecientes a la parábola $f_{1}$ (véase Gráfica 5.a).

Para el primer punto $(2,3)$

$$
\begin{aligned}
y & =a x^{2}+b x+c \\
3 & =a(2)^{2}+b(2)+c \\
3 & =4 a+2 b+c \\
4 a+2 b+c & =3
\end{aligned}
$$

Para el segundo punto $(5,0)$

$$
\begin{array}{r}
y=a x^{2}+b x+c \\
0=a(5)^{2}+b(5)+c \\
0=25 a+5 b+c \\
25 a+5 b+c=0
\end{array}
$$

Para el tercer punto $(8,3)$

$$
\begin{gathered}
y=a x^{2}+b x+c \\
3=a(8)^{2}+b(8)+c \\
3=64 a+8 b+c
\end{gathered}
$$$$
64 a+8 b+c=3
$$

Con la ecuación (5.c), (5.d) y (5.e) se plantea un sistema de ecuaciones de 3 por 3

$$
\left\{\begin{array}{l}
4 a+2 b+c=3 \\
25 a+5 b+c=0 \\
64 a+8 b+c=3
\end{array}\right.
$$

Resolviendo el sistema se obtiene que $a=\frac{1}{3}, b=\frac{-10}{3}, c=\frac{25}{3}$ de la cual se obtiene la ecuación:

$$
f_{1}=y=\frac{1}{3} \times 2-\frac{-10}{3} x+\frac{25}{3}
$$

Para $f_{2}$ 
Puntos pertenecientes a la parábola $f_{2}$ (véase Gráfica 5.a).

Para el primer punto $(4,3)$

$$
\begin{aligned}
y & =a x^{2}+b x+c \\
3 & =a(4)^{2}+b(4)+c \\
3 & =16 a+4 b+c \\
16 a+4 b & +c=3
\end{aligned}
$$

Para el segundo punto $(5,2)$

$$
\begin{aligned}
y & =a x^{2}+b x+c \\
2 & =a(5)^{2}+b(5)+c \\
2 & =25 a+5 b+c \\
25 a+5 b & +c=2
\end{aligned}
$$

Para el tercer punto $(6,3)$

$$
\begin{array}{r}
y=a x^{2}+b x+c \\
3=a(6)^{2}+b(6)+c \\
3=36 a+6 b+c \\
36 a+6 b+c=3
\end{array}
$$

Con la ecuación (5.g), (5.f) y (5.h) se plantea un sistema de ecuaciones de 3 por 3

$$
\left\{\begin{array}{l}
16 a+4 b+c=3 \\
25 a+5 b+c=2 \\
36 a+6 b+c=3
\end{array}\right.
$$

Resolviendo el sistema se obtiene que $a$ $=1, b=-10, c=27$ de la cual se obtiene la ecuación:

$$
f_{2}=y=x^{2}-10 x+27
$$

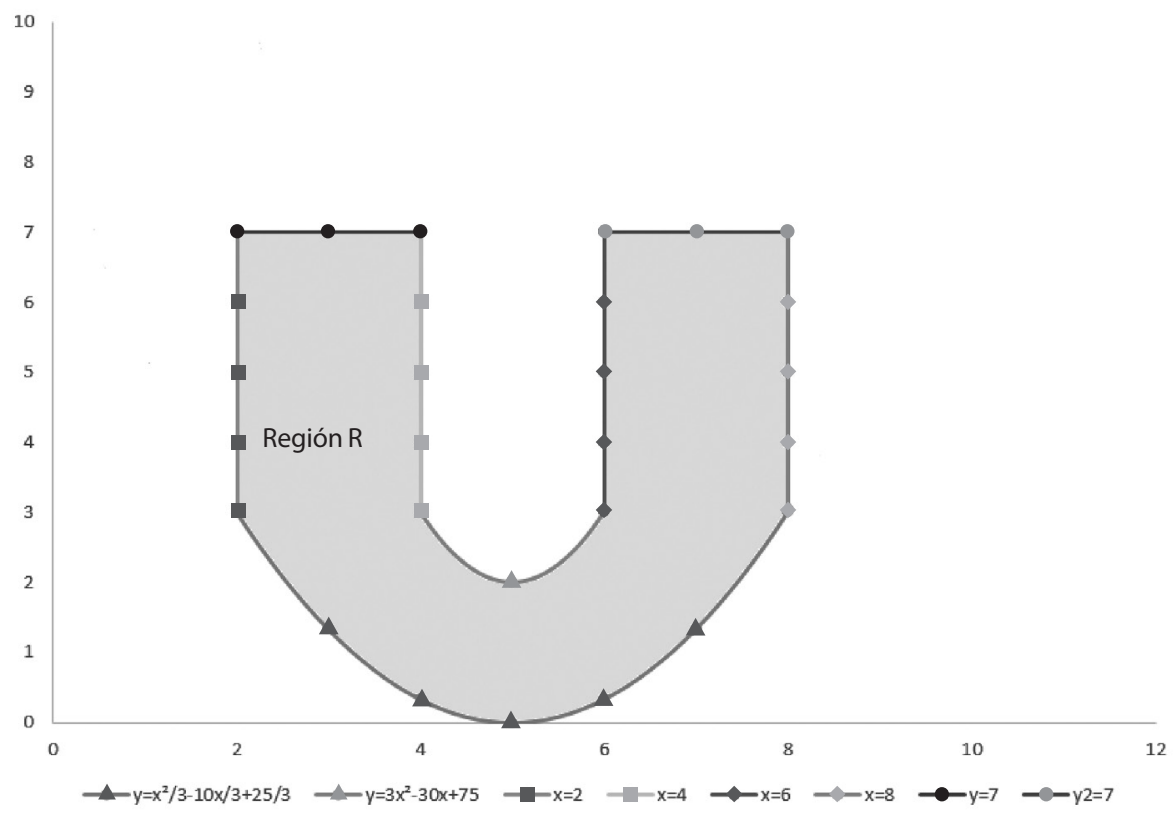

Gráfica 5.b. Ecuaciones que determinan la forma de la placa plana 
Las ecuaciones $f_{3^{\prime}}, f_{4^{\prime}} f_{5^{\prime}} f_{6^{\prime}}, f_{7}$ y $f_{8}$ representan rectas definidas entre intervalos.

En la gráfica 5.b, se observan las líneas de colores que limitan la región $R$ con sus respectivas ecuaciones.

Conociendo las ecuaciones se procede a definir la integral doble de tipo I que representa la masa de la lámina plana de la siguiente manera:

$m=\iint_{R}(1+2 x) d A=\iint_{R 1}(1+2 x) d A+\iint_{R 2}(1+2 x) d A+\iint_{R 3}(1+2 x) d A$

Con el propósito de determinar los límites de integración se trazan tres rectas que pasen por la parte inferior hasta la parte superior del plano (véase Gráfica 5.c.).
Después de haber trazado las rectas se obtienen tres regiones $R_{1}, R_{2}$, y $R_{3}$. Las regiones están determinadas por las siguientes expresiones matemáticas:

$$
\begin{gathered}
R_{1}=\left\{(x, y) \mid 2 \leq x \leq 4 \cap \frac{1}{3} x^{2}-\frac{10}{3} x+\frac{25}{3} \leq y \leq 7\right\} \\
R_{2}=\left\{(x, y) \mid 4 \leq x \leq 6 \cap \frac{1}{3} x^{2}-\frac{10}{3} x+\frac{25}{3} \leq y \leq x^{2}-10 x+27\right\} \\
R_{3}=\left\{(x, y) \mid 6 \leq x \leq 8 \cap \frac{1}{3} x^{2}-\frac{10}{3} x+\frac{25}{3} \leq y \leq 7\right\}
\end{gathered}
$$

Dada las tres expresiones anteriores se plantea tres integrales dobles, la suma de estas representan la masa de la lámina:

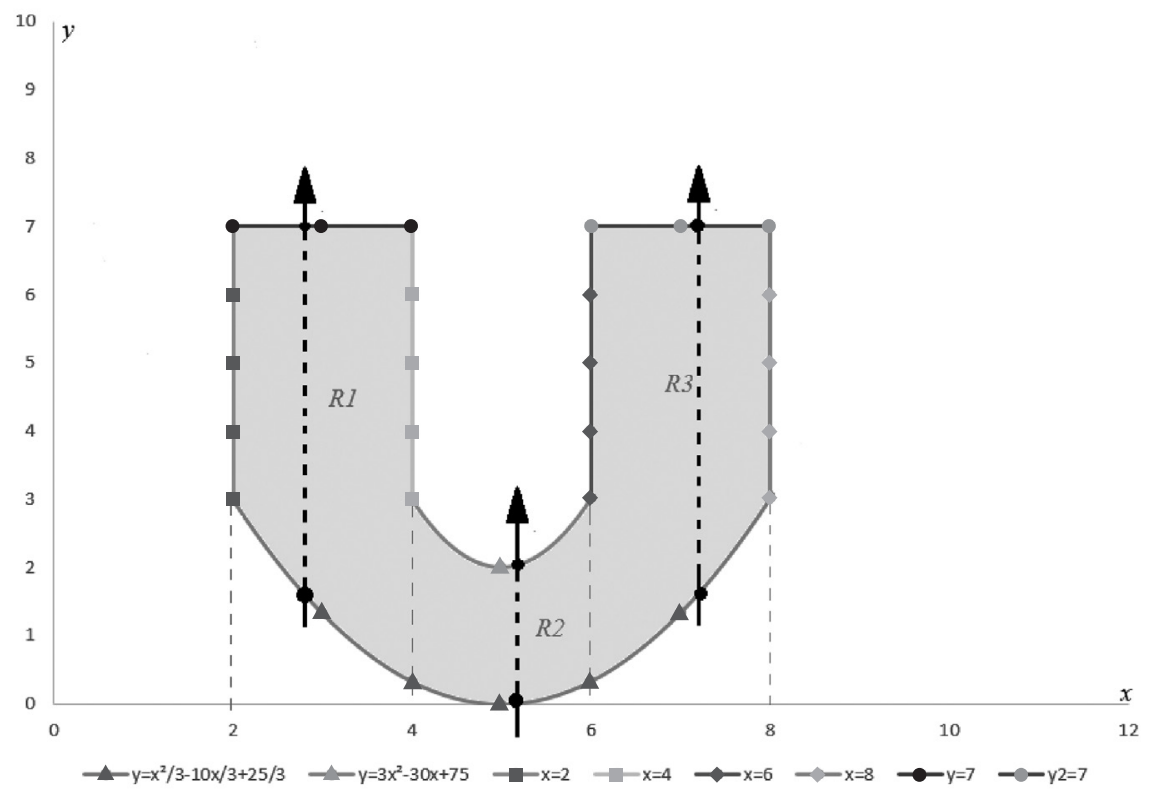

Gráfica 5.c. Límites de integración de la placa

A continuación se observa la masa de la lámina expresada en términos de tres integrables dobles.

$$
\begin{aligned}
m=\int_{2}^{4} \int_{\frac{1}{3} x^{2}-\frac{10}{3} x+\frac{25}{3}}^{7}(1+2 x) d y d x & \\
& \quad \int_{4}^{6} \int_{\frac{1}{3} x^{2}-\frac{10}{3} x+\frac{25}{3}}^{x^{2}-10 x+27}(1+2 x) d y d x+\int_{6}^{8} \int_{\frac{1}{3} x^{2}-\frac{10}{3} x+\frac{25}{3}}^{7}(1+2 x) d y d x
\end{aligned}
$$

Solución de la primer integral:

Se integra respecto a ' $y$ 'y se evalúan los límites del primer integrando

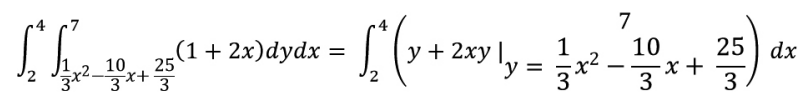


Se integra respecto a x y se evalúan los límites del segundo integrando

$$
=\int_{2}^{4}\left(7+14 x-\frac{1}{3} x^{2}+\frac{10}{3} x-\frac{25}{3}-\frac{2}{3} x^{3}-\frac{20}{3} x^{2}-\frac{50}{3} x\right) d x=
$$

El resultado de la integral que representa la región $R_{1}$ es:

$$
=\int_{2}^{4}\left(\frac{-4}{3}+\frac{2}{3} x+\frac{19}{3} x^{2}-\frac{2}{3} x^{3}\right) d x=\frac{716}{9}
$$

Solución de la segunda integral:

Se integra respecto a y y se evalúan los límites del primer integrando

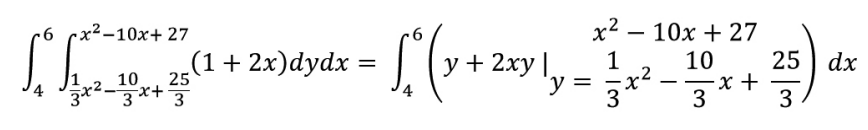

Se integra respecto a $x$ y se evalúan los límites del segundo integrando
Solución tercera integral:

Se integra respecto a y y se evalúan los límites del primer integrando

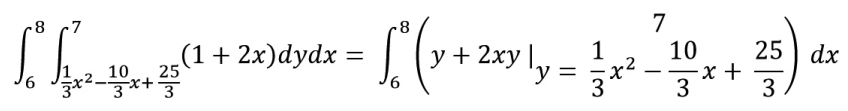

Se integra respecto a $x$ y se evalúan los límites del segundo integrando

$=\int_{6}^{8}\left(7+14 x-\frac{1}{3} x^{2}+\frac{10}{3} x-\frac{25}{3}-\frac{2}{3} x^{3}-\frac{20}{3} x^{2}-\frac{50}{3} x\right) d x=$

El resultado de la integral que representa la región $R_{3}$ es:

$$
=\int_{6}^{8}\left(\frac{-4}{3}+\frac{2}{3} x+\frac{19}{3} x^{2}-\frac{2}{3} x^{3}\right) d x=\frac{1484}{9}
$$

Obtenido los resultados de las tres integrales, es decir, de las tres regiones, la masa de la lámina es:

El resultado de la integral que representa la región $R_{2}$ es:

$$
=\int_{4}^{6}\left(\frac{56}{3}+\frac{92}{3} x-\frac{38}{3} x^{2}+\frac{4}{3} x^{3}\right) d x=\frac{440}{9}
$$




\section{Notas complementarias}

Algunas interpretaciones de la integral doble

VOLUMEN: $\operatorname{Si} f(x, y)$ es positiva sobre $R$ en el plano $X Y$, el volumen $(V)$ del sólido limitado inferiormente por $R$ y superiormente por la gráfica de $z$ $=f(x, y)$ es la integral:

$$
\operatorname{Volumen}(v)=\iint_{R} f(x, y) d A
$$

El volumen del sólido $(V)$ limitado inferiormente por la gráfica de $z=f(x, y)$ y superiormente por la de $z=g(x, y)$, para $(x, y) \in R($ región) $C R^{2}$ es la integral:

$$
\operatorname{Volumen}(v)=\iint_{R}[g(x, y)-f(x, y)] d A
$$

ÁREA: Si $R$ (región) C $R^{2}$, el área de $\mathrm{R}$ (región) es:

$$
\text { Área }(R)=\iint_{R} \mid d A
$$

MASA $^{21}$ : Si una lámina $L$ ocupa la región $R$ del plano y está compuesta por un material de densidad superficial $\rho(x, y)$, su masa es:

$$
\operatorname{Masa}(L)=\iint_{R}^{\varpi} \rho(x, y) d A
$$

DENSIDAD MEDIA: Para la lámina anteriormente descrita, la densidad de masa media es:

$$
\text { Densidad media }(L)=\frac{\iint_{R} \rho(x, y) d A}{\text { Área }(R)}
$$

21 El cálculo de masa de una región $R$, también puede emplearse para calcular la carga eléctrica $Q$, distribuida sobre una región $R . Q=\iint_{\mathrm{R}}$ $\sigma(x, y) d A$. Donde $\sigma$ es la función densidad de carga.
TEMPERATURA MEDIA: Si una lámina $L$ ocupa la región $R$ del plano y la temperatura en cada punto viene dada por $T(x, y)$, la temperatura media de la lámina es:

$$
\text { Temperatura }(L)=\frac{\iint_{R}^{\mathrm{Ei}} T(x, y) d A}{\text { Área }(R)}
$$

\section{Transformación a coordenadas polares}

Se realiza la transformada de coordenadas rectangulares a coordenadas polares cuando no es posible solucionar la integral doble por métodos convencionales.

\begin{tabular}{cc}
\hline $\begin{array}{c}\text { Coordenadas } \\
\text { rectangulares }\end{array}$ & Coordenadas Polares \\
\hline$x$ & $r \cos \theta$ \\
$y$ & $r \operatorname{sen} \theta$ \\
\hline
\end{tabular}

Tabla 1. En la tabla se observa las equivalencias entre coordenadas rectangulares y coordenadas polares.

Teniendo en cuenta la tabla anterior se tiene:

$$
\begin{gathered}
\iint_{R} f(x, y) d A=\iint_{R^{\prime}}^{\infty} f(r \cos \emptyset, r \operatorname{sen} \emptyset)\left|\frac{\partial(x, y)}{\partial(r, \theta)}\right| d r d \theta \\
R^{\prime}=\left\{(r, \theta) \mid r_{1} \leq r \leq r_{2} \cap \theta_{1} \leq \theta \leq \theta_{2}\right\} \text { Donde } 0 \leq \theta_{2}-\theta_{1} \leq 2 \pi
\end{gathered}
$$

De la expresión anterior lo único que se desconoce es $\frac{\mid(x, y)}{\partial(x, \theta)} \mid$, esta expresión recibe el nombre de Jacobiano polar.

El Jacobiano polar está definido como el determinante de las derivadas parciales de $x$ y y respecto a $r$ y $\theta$. A continuación se expresa matemáticamente:

$$
\frac{\partial(x, y)}{\partial(r, \theta)}=\operatorname{det}\left[\begin{array}{ll}
\frac{\partial x}{\partial r} & \frac{\partial x}{\partial \theta} \\
\frac{\partial y}{\partial r} & \frac{\partial y}{\partial \theta}
\end{array}\right]=\operatorname{det}\left[\begin{array}{cc}
\cos \theta & -r \operatorname{sen} \theta \\
\operatorname{sen} \theta & r \cos \theta
\end{array}\right]=r \cos ^{2} \theta+r \operatorname{sen}^{2} \theta
$$


Si se aplica la identidad fundamental $\cos ^{2} \theta$ $+\operatorname{sen}^{2} \theta=1$ se obtiene que el Jacobiano polar es igual a $r$.

$$
\frac{\partial(x, y)}{\partial(r, \theta)}=r \cos ^{2} \theta+r \operatorname{sen}^{2} \theta=r\left(\cos ^{2} \theta+\operatorname{sen}^{2} \theta\right)=r * 1=r
$$

Ecuaciones de figuras geométricas

(Espacio R3)

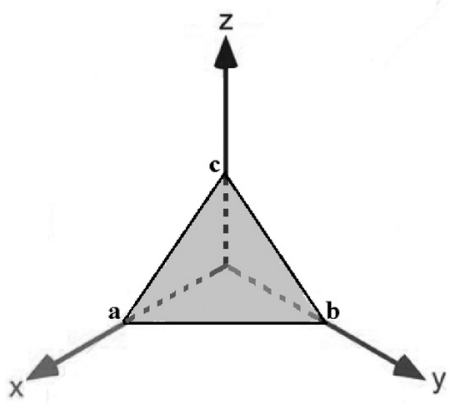

Plano:

Ecuación del plano con puntos de corte $a, b, c$ :

$$
\frac{x}{a}+\frac{y}{b}+\frac{z}{c}=1
$$

Las letras $a, b, c$ representan números reales, estos expresan los puntos de corte con los respectivos ejes $x, y, z$.

En el caso de que alguna de las variables $x, y$, $z$ no apareciera en la ecuación significaría que el plano no corta a dicho eje..22

Por ejemplo, en la Figura 6 se tiene un plano que no corta al eje $z$, es decir, es paralelo al eje $z$. En la Figura 7, se observa un plano que no corta a los ejes $x$ y $y$, por lo tanto, es paralelo al plano $x y$ :

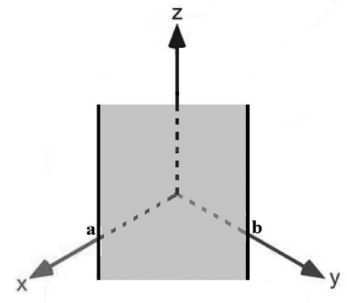

Figura 1

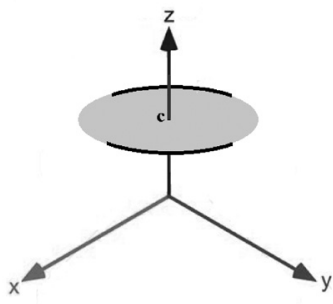

Figura 2
Las ecuaciones que representan a las Figura 1 y 2 respectivamente son: $\frac{x}{a}+\frac{y}{b}=1$ y $\frac{z}{c}=1$

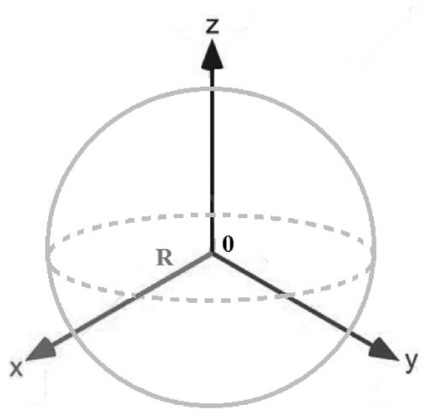

\section{Esfera:}

Ecuación de la esfera centrada en el origen 0:

$$
x^{2}+y^{2}+z^{2}=R^{2}
$$

$R$ representa el radio de la esfera centrada en el origen $\mathbf{0}$.

Ecuación de la esfera centrada en un punto $\mathbf{P}(a, b, c)$ :

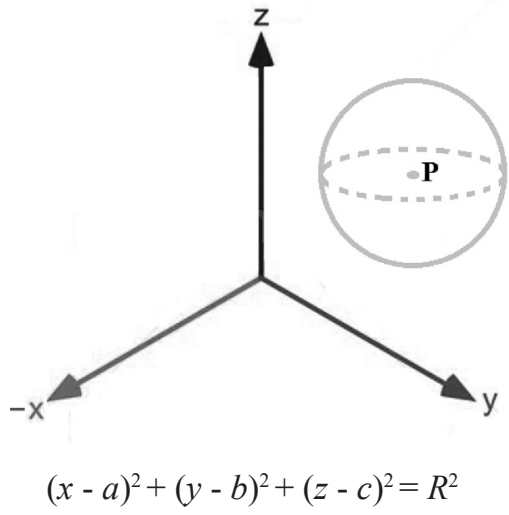

22 Cuando se menciona que no corta a dicho eje se quiere decir que realmente lo corta en el infinito. 


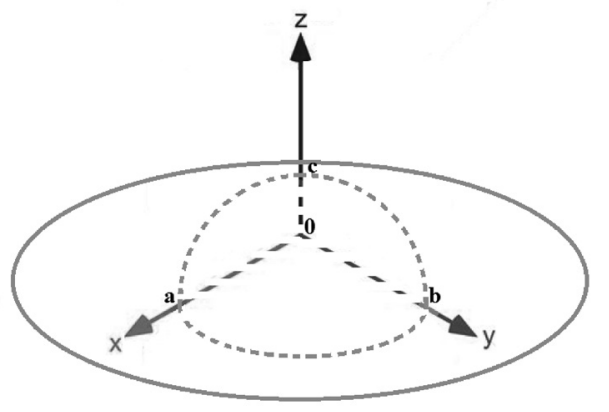

\section{Elipsoide:}

Ecuación del elipsoide centrada en el origen 0:

$$
\frac{x^{2}}{a^{2}}+\frac{y^{2}}{b^{2}}+\frac{z^{2}}{c^{2}}=1
$$

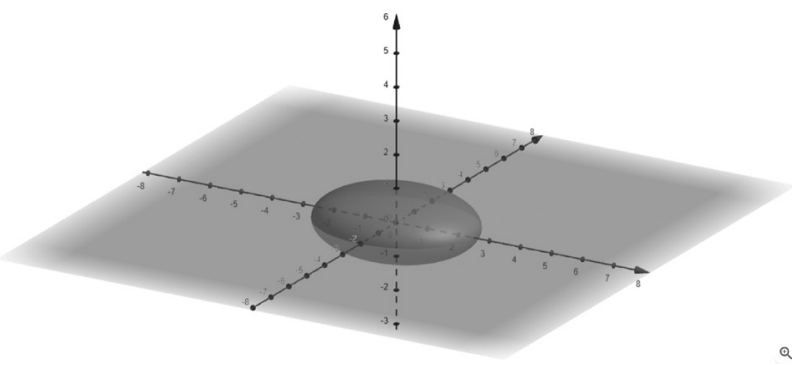

Las letras $a, b, y$ c representan los semiejes de las secciones elípticas.

En la gráfica del elipsoide se asignó los siguientes valores:

$$
a=2.5, b=2, c=1.5
$$

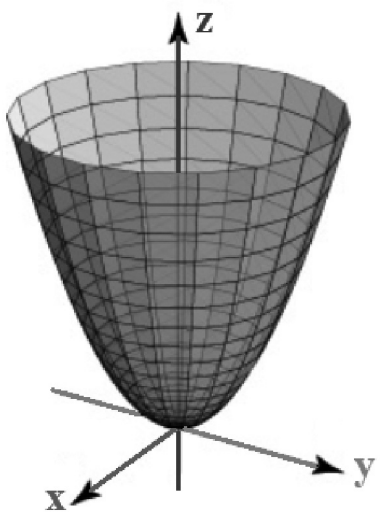

\section{Paraboloide circular:}

Ecuación del paraboloide circular:

$$
z=x^{2}+y^{2}
$$

Las secciones transversales al eje $Z$ son circulares.

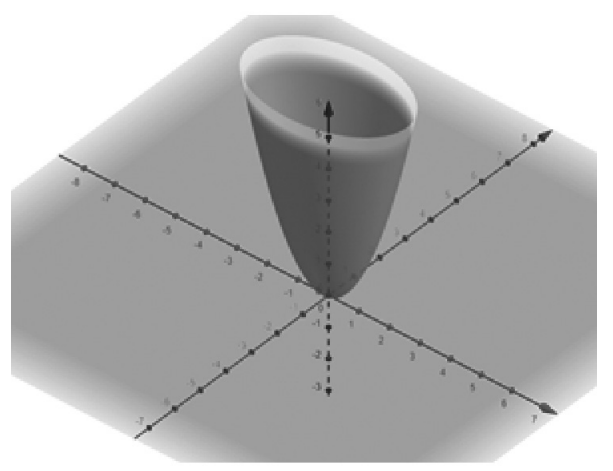

\section{Paraboloide Elíptico:}

Ecuación del paraboloide Elíptico:

$$
z=m x^{2}+n y^{2}
$$

Las secciones transversales al eje $z$ son elípticas.

En la gráfica del paraboloide elíptico:

$$
m>n
$$

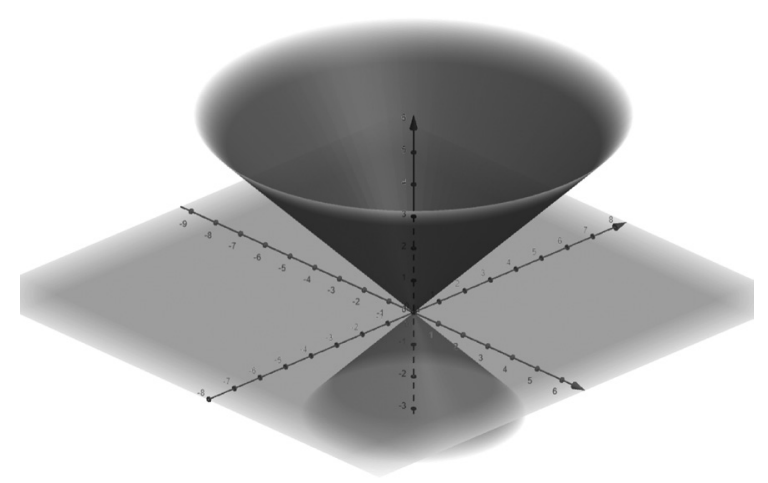

\section{Superficie cónica:}

\section{Superficie cónica circular}

Ecuación de la superficie cónica circular:

$$
z^{2}=x^{2}+y^{2}
$$

Las secciones transversales al eje $z$ son circulares. 


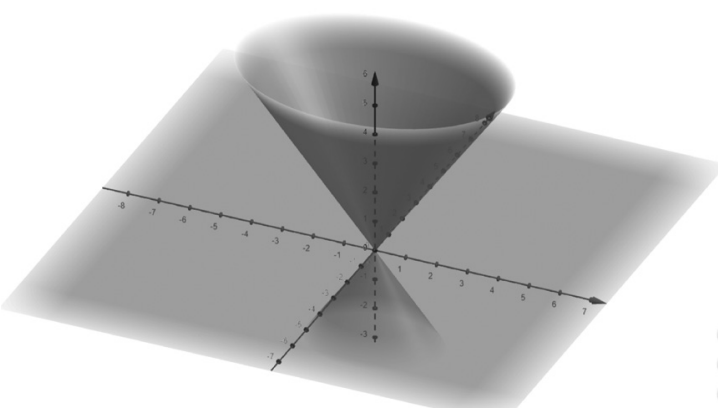

Superficie cónica elíptica:

Ecuación de la superficie cónica elíptica:

$$
z^{2}=m x^{2}+n y^{2}
$$

Las secciones transversales al eje z son elípticas.

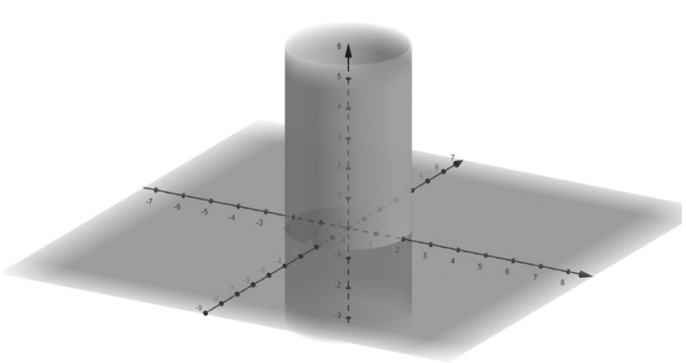

\section{Superficie cilíndrica:}

Ecuación de la superficie cilíndrica circular:

$$
x^{2}+y^{2}=R^{2}
$$

Las secciones transversales al eje $z$ son circulares.

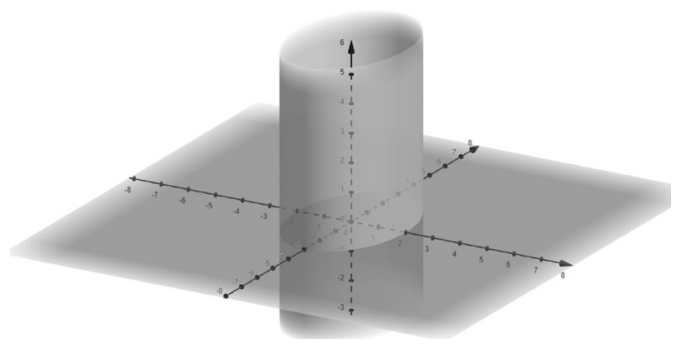

\section{Cilindroide:}

Ecuación del cilindroide:

$$
\frac{x^{2}}{a^{2}}+\frac{y^{2}}{b^{2}}=1
$$

Las secciones transversales al eje $z$ son elipses de semiejes $a, b$.

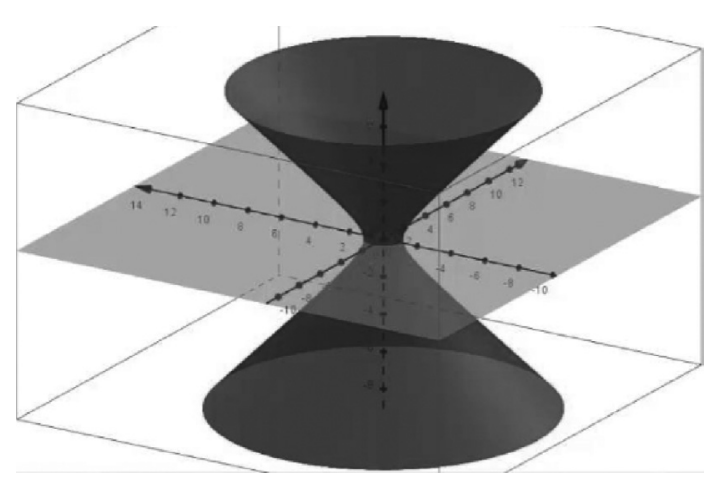

\section{Hiperboloide de una hoja:}

$$
-\frac{x^{2}}{a^{2}}+\frac{y^{2}}{b^{2}}+\frac{z^{2}}{c^{2}}=1
$$

Si $b=c$ se trata de un hiperboloide de revolución.

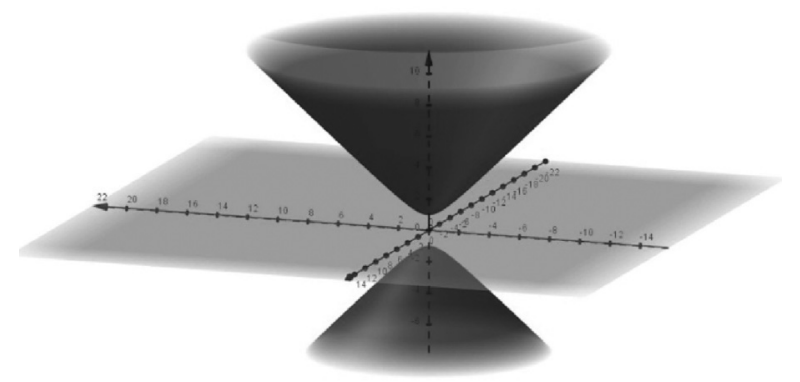

Hiperboloide de dos hojas:

$$
-\frac{x^{2}}{a^{2}}+\frac{y^{2}}{b^{2}}+\frac{z^{2}}{c^{2}}=1
$$

Método de Riemann para integrales dobles

A continuación, se desarrolla paso a paso una integral doble mediante la doble suma de Riemann.

Para aplicar el método de Riemann los límites de la integral deben estar definidos por números. 
Si se analiza previamente la integral doble se puede observar que el área a tener en cuenta representa un rectángulo de base 2 y altura 1 .

$$
\int_{1}^{2} \int_{1}^{3}\left(x^{2}-3 y\right) d x d y
$$

Se aplica el método de Riemann reemplazando $\lim _{n \rightarrow 0} \lim _{k \rightarrow 0}$ por $\lim _{p \rightarrow \infty}$ este cambio se hace con el fin de facilitar la escritura en el desarrollo de las siguientes sumatorias.

$$
R_{i}=\lim _{p \rightarrow \infty} \sum_{j=1}^{n} \sum_{i=1}^{k} f\left(x_{i}^{\prime}, y_{j}^{\prime}\right) \Delta x_{i} \Delta y_{j}
$$

A partir de ahora se determina a qué es equivalente cada expresión que conforma la ecuación canónica del método de Riemann 6.a.

$$
\Delta x_{i}=\frac{b-a}{k}=\frac{3-1}{k}=\frac{2}{k} \quad \Delta y_{j}=\frac{d-c}{n}=\frac{2-1}{n}=\frac{1}{n}
$$

Después de hallar el valor de $\Delta x_{\mathrm{i}} \mathrm{y} \Delta \mathrm{y}_{\mathrm{j}}$ se sustituye $x_{i}^{\prime}=1+\frac{2 i}{k}$ y $y_{j}^{\prime}=1+\frac{1 j}{n}$ teniendo en cuenta que el intervalo empieza en 1 y va sumando $1+$ $\frac{2 i}{k}$ para $x_{i}^{\prime} \mathrm{y} 1+\frac{1 j}{n}$.

$$
R_{i}=\lim _{p \rightarrow \infty} \sum_{j=1}^{n} \sum_{i=1}^{k} f\left(1+\frac{2 i}{k}, 1+\frac{1 j}{n}\right) \frac{2}{k} * \frac{1}{n}
$$

Después de eso evaluamos $x_{i}^{\prime} y y_{j}^{\prime}$ en la función $x^{2}-3 y, x_{i}^{\prime}=x$ y $y_{j}^{\prime}=y$.

$$
R_{i}=\lim _{p \rightarrow \infty} \sum_{j=1}^{n} \sum_{i=1}^{k}\left(\left(1+\frac{2 i}{k}\right)^{2}-3\left(1+\frac{1 j}{n}\right)\right) \frac{2}{k n}
$$

Se desarrollan los binomios con el propósito de desarrollar las sumatorias

$$
R_{i}=\lim _{p \rightarrow \infty} \sum_{j=1}^{n} \sum_{i=1}^{k}\left(1+\frac{4 i}{k}+\frac{4 i^{2}}{k^{2}}-3-\frac{3 j}{n}\right) \frac{2}{k n}
$$

Aplicando propiedades de linealidad separamos en 5 sumatorias para facilitar su desarrollo

$$
\begin{gathered}
R_{i}=\lim _{p \rightarrow \infty} \frac{2}{k n} \sum_{j=1}^{n}\left(\sum_{i=1}^{k} 1+\sum_{i=1}^{k} \frac{4 i}{k}+\sum_{i=1}^{k} \frac{4 i^{2}}{k^{2}}-\sum_{i=1}^{k} 3-\sum_{i=1}^{k} \frac{3 j}{n}\right) \\
R_{i}=\lim _{p \rightarrow \infty} \frac{2}{k n} \sum_{j=1}^{n}\left(k+\frac{4}{k} \frac{k(k+1)}{2}+\frac{4}{k^{2}} \frac{k(k+1)(2 k+1)}{6}-3 k-\frac{3 j k}{n}\right) \\
R_{i}=\lim _{p \rightarrow \infty} \frac{2}{k n} \sum_{j=1}^{n}\left(k+2(k+1)+\frac{2}{3 k}(k+1)(2 k+1)-3 k-\frac{3 j k}{n}\right)
\end{gathered}
$$

Resuelta la sumatoria con índice $i$ se procede a hacer lo mismo ahora con respecto a la sumatoria con índice $j$.

$$
\begin{gathered}
R_{i}=\lim _{p \rightarrow \infty} \frac{2}{k n}\left(k \sum_{j=1}^{n} 1+2(k+1) \sum_{j=1}^{n} 1+\frac{2}{3 k}(k+1)(2 k+1) \sum_{j=1}^{n} 1-3 k \sum_{j=1}^{n} 1\right. \\
\left.-\frac{3 k}{n} \sum_{j=1}^{n} j\right) \\
R_{i}=\lim _{p \rightarrow \infty} \frac{2}{k n}\left(k n+2 n(k+1)+\frac{2 n}{3 k}(k+1)(2 k+1)-3 k n-\frac{3 k n}{n} \frac{n(n+1)}{2}\right) \\
R_{i}=\lim _{p \rightarrow \infty} \frac{2}{k n}\left(k n+2 n k+2 n+\frac{2 n}{3 k}\left(2 k^{2}+k+2 k+1\right)-3 k n-\frac{3 k n}{2}-\frac{3 k}{2}\right)
\end{gathered}
$$

Simplificamos las expresiones matemáticas

$$
R_{i}=\lim _{p \rightarrow \infty} \frac{2}{k n}\left(2 n+\frac{4 k^{2} n}{3 k}+\frac{2 k n}{3 k}+\frac{4 k n}{3 k}+\frac{2 n}{3 k}-\frac{3 k n}{2}-\frac{3 k}{2}\right)
$$

$$
R_{i}=\lim _{p \rightarrow \infty} \frac{2}{k n}\left(2 n+\frac{4 k n}{3 k}+\frac{2 n}{3}+\frac{4 n}{3}+\frac{2 n}{3 k}-\frac{3 k n}{2}-\frac{3 k}{2}\right)
$$

Luego de haber operado las sumatorias se halla el límite de $n$ y $k$ implícitos en $p$ cuando tienden $a \infty$.

$$
\begin{gathered}
R_{i}=\lim _{p \rightarrow \infty}\left(\frac{4}{k}+\frac{8}{3}+\frac{4}{3 k}+\frac{8}{3 k}+\frac{4}{3 k^{2}}-3-\frac{3}{n}\right) \\
R_{i}=0+\frac{8}{3}+0+0+0-3+0=\frac{-1}{3}
\end{gathered}
$$


Finalmente, se obtiene que la integral $\int_{1}^{2} \int_{1}^{3}\left(x^{2}-3 y\right) d x d y$ es igual a $\frac{-1}{3}$.

El lector podrá comprobar la respuesta solucionando la integral doble de manera directa. Es decir, integrando respecto a $x$ y a $y$.

\section{Referencias}

Apostol, T. M. (2001). Cálculo con funciones de varias variables y álgebra lineal, con aplicaciones a las ecuaciones diferenciales y a las probabilidades (vol. II). Barcelona: Reverté.

Cisneros, G. (2012). Integrales múltiples y sus aplicaciones. Universidad de Carabobo. Departamento de Matemáticas.

Duval, R. (1992). Gráficas y ecuaciones: la articulación de dos registros. En R. Cambray, E. Sánchez y G. Zubieta, Antología en educación matemática, material de apoyo para el seminario de educación matemática 1. Maestría en Ciencias, Especialidad en Matemáticas (pp. 125-141). Bogotá: CINESTAV-IPN.

Gómez, P. (2004). Análisis didáctico y uso de tecnología en el aula de matemáticas. En M. Peñas, A. Moreno y J. L. Lupiáñez, Investigación en el aula de matemáticas: tecnologías de la información y la comunicación (pp. 73-95). Universidad de Granada. Investigación en el aula de matemáticas: tecnologías de la información y la comunicación SAEM Thales - Departamento de Didáctica de la Matemática.

Guzman, M. D. (1 de Abril de 2017). Tendencias innovadoras en educación matemática. Universidad Complutense de Madrid. Recuperado de http://www.mat. ucm.es/catedramdeguzman/drupal/ migueldeguzman/legado/educacion/ tendenciasInnovadoras

Salas, S., Hille, E. y Etgen, G. (2002). Calculus; una y varias variables (vol. II). Barcelona: Reverté. 


\section{Pre-Impresos 14}

\section{Eventos de la Facultad}

\section{$9^{\circ}$ Congreso Nacional de Enseñanza de la Física y la Astronomía}

Este espacio de reflexión sobre la investigación en enseñanza de la Física y la Astronomía contará con invitados nacionales e internacionales, y en él se abordarán temas como la historia y la epistemología de la Física y la Astronomía, el uso de las TIC, la experimentación y la didáctica en el aula, todo en relación con la enseñanza de estas dos disciplinas en distintos niveles educativos y en diferentes contextos culturales.

Al respecto dialogamos con la profesora Rosa Nidia Tuay del Departamento de Física de la Universidad Pedagógica Nacional y miembro del Comité Organizador del $9^{\circ}$ Congreso Nacional de Enseñanza de la Física y la Astronomía (CNEFA).

Pre-Impresos: ¿Cuáles son los antecedentes e historia del CNEFA?

Rosa Nidia Tuay (NT): El congreso en sus diferentes versiones busca convocar a la comunidad académica de todo el país. Se ha realizado en dos ocasiones en la ciudad de Bogotá con el apoyo de las Universidades Pedagógica y Distrital, y también en la Universidad del Tolima en Ibagué, en la Universidad de Antioquia en Medellín, en la Universidad del Valle en Cali, en la Universidad Tecnológica de Pereira y en la Universidad de Nariño en San Juan de Pasto.

Entre sus líneas de acción está discutir aspectos importantes acerca del estado de la enseñanza de las ciencias en Colombia, específicamente de la Física y al Astronomía, a partir de los diferentes trabajos, artículos y tesis publicados por agentes que pertenecen a al comunidad de educadores en Física y Astronomía, y que son el resultado de innovaciones e investigaciones en torno a las diversas problemáticas que surgen en el ámbito educativo de la Física en Colombia. Y aunque estos aspectos nos brindan un panorama de lo que se hace en enseñanza y didáctica de las ciencias, al parecer los procesos entorno a estos no son suficientes, y hoy en día bajo diversos puntos de vista se considera que la enseñanza de las ciencias en nuestro país está en un proceso de consolidación y delimitación de sus propias problemáticas y líneas de investigación, las cuales deben ir enfocadas en el pensamiento de nuevos enfoques pedagógicos que permitan el diálogo constructivo con tendencias internacionales y generen identidad nacional al ser pensados entorno a nuestras problemáticas actuales de orden social y cultural.
Pre-Impresos: ¿Qué tipo de evento es el 9० Congreso Nacional de Enseñanza de la Física y la Astronomía (CNEFA)? ¿En qué consiste?

NT: El $9^{\circ}$ Congreso Nacional de Enseñanza de la Física y la Astronomía es un espacio de encuentro para reflexionar sobre la investigación en enseñanza de la Física y la Astronomía en Colombia, considerando las condiciones de diversidad cultural, propias de los contextos en donde se desarrolla, en diálogo con las perspectivas internacionales en esta materia.

Pre-Impresos: ¿Qué finalidad tiene el evento y a quién está dirigido?

NT: Este evento reúne docentes de Física de todos los niveles educativos, investigadores en enseñanza de la Física, entes gubernamentales y empresariales relacionados directamente con la educación; todos interesados en compartir, reflexionar y discutir en torno a propuestas, prácticas, nuevos enfoques, estrategias y teorías de la problemática educativa de la enseñanza y el aprendizaje de la Física que se plantean en los diferentes contextos educativos del país.

Pre-Impresos: ¿Cuáles son los aspectos para destacar del $9^{\circ}$ CNEFA?

NT: En el marco de esta versión del congreso, tendrá lugar el simposio "Historiadoras de las ciencias, aportes a la formación de licenciados en Física", escenario para la construcción de alternativas y fortalecimiento de los procesos de renovación de los programas de licenciatura en Física.

En el congreso se manifiesta un variado espectro de opciones, reflexiones, propuestas, iniciativas e investigaciones y cuenta con la participación de destacados invitados de orden nacional e internacional que se constituyen en uno de los centros de interés de la dinámica académica que se vive durante su realización.

Pre-Impresos: ¿Quiénes son los organizadores del congreso?, ¿cada cuánto se realiza?, y ¿qué duración tiene?

NT: Las entidades convocantes son la Universidad Pedagógica Nacional, la Universidad Distrital Francisco José de Caldas y la Asociación Colombiana de profesores de Física. Cuenta con el apoyo de universidades internacionales como la Universidad de Salta y la Universidad Santiago de Chile, de universidades colombianas como la Universidad Antonio Nariño, la Universidad del Valle y la Universidad de Nariño. Asimismo, cuenta con del 
apoyo de entidades territoriales como la Secretaría de Educación de Bogotá, el IDEP y la Secretaría de Educación de Cundinamarca. Además de entidades privadas como la Fundación Francisca Radke.

El congreso se celebra cada dos años y esta versión se realizará los días 7, 8 y 9 de noviembre de 2018 en Bogotá, en las instalaciones de la Universidad Antonio Nariño, sede Nicolás de Federman.

Pre-Impresos: ¿Cuál es la importancia del evento para la comunidad educativa de maestros y estudiantes de (licenciatura en) ciencias?

NT: Los logros, dificultades, obstáculos y elaboraciones de la comunidad permiten evidenciar líneas de inves- tigación que definen y caracterizan la comunidad de educadores en Física, a partir de fundamentos históricos y epistemológicos de la enseñanza, el aprendizaje y la didáctica de la Física, así como también entrar a revisar los fundamentos de prácticas, procesos y metodologías de investigación.

Pre-Impresos: ¿Cuáles son las proyecciones a corto, mediano y largo plazo y cuándo será la próxima versión?

NT: Continuar la reflexión sobre la enseñanza de la Física y la Astronomía, asunto que es tarea fundamental en los procesos de formación, asimismo, se proyecta realizar la décima versión del Congreso en el año 2020 en la Universidad de la Amazonía en la ciudad de Florencia, Caquetá. 


\section{Pre-Impresos 14}

\section{ACERCA DE LA SERIE PRE•IMPRESOS}

La serie Pre./mpresos Estudiantes es un proyecto de la Facultad de Ciencia y Tecnología (FCT) de la Universidad Pedagógica Nacional que divulga a través de la comunicación escrita la producción intelectual de los autores, destacando sus experiencias y reflexiones respecto de los temas inherentes a sus campos disciplinares específicos y su enseñanza. Por tanto, configura un espacio de visibilidad y reconocimiento público del trabajo de los maestros en formación y en ejercicio adscritos a la FCT.

La escritura en el ámbito de las ciencias y la tecnología

La comunicación es un aspecto fundamental de los procesos de cognición que construye relaciones de fuerza e identificación entre las personas y define el lugar de cada individuo en un grupo. Así, toda relación social se funda en el intercambio de ideas, pues cuando hablamos y escribimos también damos forma al mundo. Por tanto, la conformación de comunidades académicas tiene un carácter social y comunicativo, proceso en el que la palabra escrita contribuye a la socialización de las ideas; dado que, la comunicación de la ciencia se realiza en lengua natural.

¿Qué es un impreso?

Los Pre-impresos son una publicación previa que se utilizan en comunidades académicas para difundir el trabajo de sus miembros y contribuir a la formación de futuros investigadores, apoyando la cualificación de sus procesos escriturales.

\section{Origen}

Este proyecto editorial también constituye un espacio académico de formación y cualificación docente, que se inspiró en un trabajo similar que realiza el grupo Física y Cultura del Departamento Física de la FCT, con trabajos de profesores, desde principios de la década de 1990, con el fin de promover la circulación de las ideas de los profesores adscritos a este grupo de investigación.

\section{Objetivos}

Pre.Impresos Estudiantes promueve el fortalecimiento de la actividad académica en dos dimensiones; como proceso de formación escritural de los futuros maestros de ciencias, matemática y tecnología, y como iniciativa editorial que se traduce en una publicación seriada que divulga la producción intelectual de los estudiantes de la FCT.
El carácter del proceso realizado y el acompañamiento escritural que se brinda desde el proyecto hacen de esta experiencia una actividad académica de formación docente, con proyección en la práctica pedagógica e investigativa que contribuye a:

- Apoyar los fines misionales de la Universidad de investigar, producir y difundir conocimiento profesional docente, educativo, pedagógico y didáctico, además de propiciar una interacción con la sociedad para aportar a la construcción de nación.

- Propiciar una mayor consciencia lingüística, al poner de relieve la relación entre ciencia y lenguaje en el proceso de construcción textual, que requiere el desarrollo de la capacidad discursiva y habilidades comunicativas.

- Fortalecer la comunidad académica de la Facultad, al visibilizar las líneas de trabajo de los grupos de investigación de las diferentes unidades académicas.

\section{Características}

Pre./mpresos Estudiantes es un proyecto institucional de carácter extra curricular en el que pueden participar los estudiantes de los diferentes programas de la Facultad que quieran vincularse, ya sea, de manera individual o en grupo. El proceso de acompañamiento que se brinda exige compromiso y disciplina de los participantes, para la cualificación de su proceso escritural. Los temas a trabajar pueden cobijar una amplia gama de aspectos relacionados con las disciplinas -las ciencias, la matemática, la tecnología- y su enseñanza, así como, con la educación en general, ya sean reflexiones de carácter epistemológico y/o pedagógico, entre otras posibilidades.

Se puede participar con un amplio tipo de formatos de escritura, como por ejemplo: artículos, ponencias, módulos didácticos, cartillas, ensayos, crónicas, experiencias de aula, diarios, informes de investigación, por solo mencionar algunos. El proceso de elaboración, edición y publicación final de cada documento se ajusta al tiempo requerido por los autores para culminar esta labor. La publicación se hace en forma de cuadernillos monográficos en formato digital e impreso. La convocatoria es permanente. 


\section{Sobre el autor}

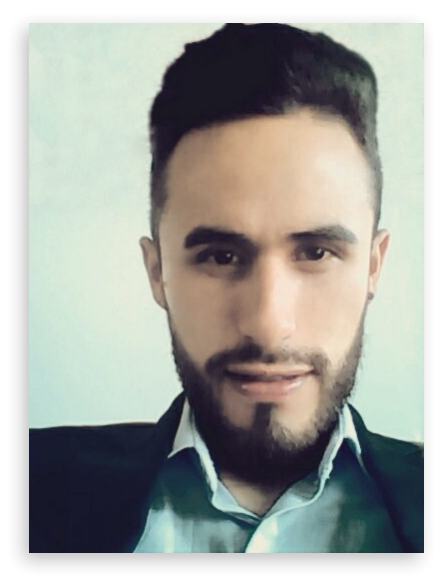

Luis Fernando Maldonado Osorio, estudiante de la Licenciatura en Electrónica de la Universidad Pedagógica Nacional. Realizó estudios en Diseño e Integración de Automatismos Mecatrónicos (SENA, 2011). Campeón en la categoría Kirchhoff del torneo The King of Circuit, orientado a medir la habilidad en el análisis y desarrollo de circuitos, organizado por la Universidad Pedagógica Nacional (mayo, 2013). Obtuvo el primer puesto, categoría seguidor de línea, en el Torneo Nacional de Robótica 2013, realizado en la regional SENA de Ibagué. Participó como miembro del equipo de docentes y estudiantes de la Licenciatura en electrónica de la uPN en el reto de innovación IEEE 2018, CANSAT - Colombia para la paz, convocatoria encaminada al diseño mecatrónico de un mini-satélite para medir las variables: temperatura, presión atmosférica, humedad y altitud, entre otras, en zonas urbanas y rurales del país. Ha sido monitor académico en las áreas de matemáticas, circuitos, diseño digital, señales y sistemas; entre sus intereses está el análisis, la construcción y el desarrollo de herramientas didácticas para facilitar la comprensión de diferentes aspectos relacionados con la mecatrónica. Sus grandes pasiones son la docencia y el conocimiento aplicado a situaciones de la vida real.

\section{http://revistas.pedagogica.edu.co}

